\title{
Model spin-orbit coupling Hamiltonians for graphene systems
}

\author{
Denis Kochan, Susanne Irmer, and Jaroslav Fabian \\ Institute for Theoretical Physics, University of Regensburg, 93040 Regensburg, Germany
}

(Received 27 October 2016; published 12 April 2017)

\begin{abstract}
We present a detailed theoretical study of effective spin-orbit coupling (SOC) Hamiltonians for graphene-based systems, covering global effects such as proximity to substrates and local SOC effects resulting, for example, from dilute adsorbate functionalization. Our approach combines group theory and tight-binding descriptions. We consider structures with global point group symmetries $D_{6 h}, D_{3 d}, D_{3 h}, C_{6 v}$, and $C_{3 v}$ that represent, for example, pristine graphene, graphene miniripple, planar boron nitride, graphene on a substrate, and free standing graphone, respectively. The presence of certain spin-orbit coupling parameters is correlated with the absence of the specific point group symmetries. Especially in the case of $C_{6 v}$ - graphene on a substrate, or transverse electric field-we point out the presence of a third SOC parameter, besides the conventional intrinsic and Rashba contributions, thus far neglected in literature. For all global structures we provide effective SOC Hamiltonians both in the local atomic and Bloch forms. Dilute adsorbate coverage results in the local point group symmetries $C_{6 v}, C_{3 v}$, and $C_{2 v}$, which represent the stable adsorption at hollow, top and bridge positions, respectively. For each configuration we provide effective SOC Hamiltonians in the atomic orbital basis that respect local symmetries. In addition to giving specific analytic expressions for model SOC Hamiltonians, we also present general (no-go) arguments about the absence of certain SOC terms.
\end{abstract}

DOI: 10.1103/PhysRevB.95.165415

\section{INTRODUCTION}

The ability to synthesize, manipulate, and functionalize 2D materials is an ultimate milestone in technological development and current fundamental research, including spintronics $[1,2]$. One of the major challenges is controlling, engineering, and harvesting spin degrees of freedom for faster data processing, storage, etc. Graphene seems to be a promising material [3] for such applications due to its high bipolar mobility [4], chemical and mechanical [5] stability, "relativistic" band structure [6] with chiral electrons that are highly insensitive to backscattering [7,8], and, importantly for spintronics, weak intrinsic spin-orbit coupling (SOC) [9]. The latter was theoretically estimated [10-14] to yield long spin lifetimes-orders of microseconds-enough for harvesting electron spins as "carriers of information." However, experiments carried out on graphene devices of the first generation gave spin lifetimes three order of magnitudes smaller [15-21]. This vast discrepancy can be reliably explained assuming a small amount (orders of ppm) of resonant magnetic scatters [22-24] like for example hydrogen atoms [25,26] or vacancies [26,27]. Related theoretical studies [28-30] confirmed that magnetic moments, indeed, strongly affect spin dynamics and can cause the ultra-fast spin relaxation. A recent experiment of the Valenzuela group [31], analyzing graphene's spin-lifetime anisotropy, supports that view and convincingly rules out SOC as a determining factor of the fast spin relaxation.

On the other hand, enhancing SOC in graphene is desirable as well. Indeed, graphene with strong intrinsic SOC is predicted to host the quantum spin Hall phase [32]. Therefore, one of the current technological and theoretical challenges is to tailor the strength of SOC of graphene in a controllable manner. In fact, SOC can be significantly enhanced either by chemical functionalization - coating of graphene with light [33-39] or heavy [40-43] adatoms - accompanied by band gap opening,

*denis.kochan@physik.uni-regensburg.de curvature as in the case of carbon nanotubes [44,45], or by a variety of proximity effects resulting from substrates or due to scaffolding of different 2D materials [46]. Tangible examples are CVD graphene grown on $\mathrm{Cu}$ and $\mathrm{Ni}$ substrates [47-49], or graphene placed on top of transition metal dichalcogenides [50-53].

To further examine SOC effects in functionalized graphene and also design device properties, one needs an effective model that allows reliable simulations of the spin and charge transport characteristics [28,54-68]. In this paper, we present a detailed symmetry analysis focusing on effective SOC Hamiltonians in a way that is complementary to Refs. [40,58,69]. Our findings remain valid for any hexagonal (graphenelike) structure possessing $\pi$ orbitals and are easily transferable to other systems. The primary aim of this manuscript is to lift the curtain and show practically how to derive the corresponding SOC Hamiltonians from the given pools of global or local symmetries.

We discuss two cases: global SOC Hamiltonians that represent proximity induced phenomena or periodically functionalized structures, and local SOC Hamiltonians that govern spin dynamics in the vicinity of adsorbates. Starting with pristine graphene, we step-by-step reduce the number of global symmetries approaching structures such as graphene miniripple, staggered graphene, planar boron nitride, silicene, graphene on a substrate, graphone, etc. For each representative case, which is classified by the associated subgroup of the full hexagonal group, we derive an effective SOC Hamiltonian in real and reciprocal spaces, respectively. Our analysis therefore covers also quasimomenta that are not necessarily constrained to the vicinity of Dirac points.

In the case of local impurities, we focus on the reduction of local symmetries up to a certain spatial extent from the adsorbate. The three representative adsorption positions are hollow, top, and bridge and we provide here the local SOC Hamiltonians in real space. Group arguments allow us to link the presence or absence of certain symmetries to various spinorbit couplings that emerge in the effective SOC Hamiltonian. For example, in the global case corresponding to point group 
$C_{6 v}$ - graphene in a transverse electric field or deposited on a substrate-we highlight the presence of a SOC term that have not yet been considered. It appears along with the conventional intrinsic and Rashba couplings and is related to the absence of the principal mirror plane in the structure.

The paper is organized as follows. After recapitulating the basic group theory related with the full hexagonal system and its application to SOC matrix elements in Sec. II, we discuss separately translational invariant systems, Sec. III, and systems lacking that invariance (local adsorbates), Sec. IV. In sections of III, we cover in detail SOC in pristine graphene, point group $D_{6 h}$, and effective SOC Hamiltonians in systems that are characterized by one of its subgroups: $D_{3 d}, D_{3 h}, C_{6 v}$, and $C_{3 v}$. Section IV is devoted to local SOC Hamiltonians for the three stable adsorption positions-hollow, top, and bridge, respectively. Summary and final remarks are provided in Sec. V.

\section{GROUP THEORY AND SOC: PRELIMINARIES}

A convenient approach how the group theory enters effective model building is a decomposition of the Hamiltonian matrix elements associated with the problem into irreducible representations (irreps). Those are well known and standardly tabulated for all crystallographic point groups [70,71]. Considering spin and spin-orbit interaction the irrep analysis around the high-symmetry points in the Brillouin zone becomes more involved. This is because the associated double (also called spinor) group representations should be appropriately taken into account; the case of graphite is exhaustively discussed in the thesis of Slonczewski [72]. For a general overview and connection with the theory of group invariants, see the book of Bir and Pikus [73], or Winkler [74].

Another possibility how to derive an effective SOC Hamiltonian is to employ the multi-orbital tight-binding approach [75-78]. The group symmetry analysis on the orbital level is straightforward and well described by the Koster-Slater two-center approximation [79] and, consequently, SOC enters as the intra-atomic LS interaction $\xi_{\ell} \hat{\mathbf{L}} \cdot \hat{\mathbf{S}}$. The resulting multiorbital tight-binding Hamiltonian is then downfolded by means of the Löwdin projection [80] to the states of interest-mostly the low-energy states with respect to the Fermi level.

As an alternative to the invariant expansion and the multiorbital tight-binding method with the Löwdin projection, we present here an effective tight-binding approach that employs symmetries of local atomic orbitals. We focus particularly on hexagonal lattice structures assuming the low-energy physics near the Fermi level can be approximately well described by $\pi$ orbitals, i.e., carbon $2 p_{z}$ orbitals, or atomic orbitals $n, \ell \neq$ $0, m_{\ell}=0$. For simplicity, we consider that each nodal atomic site $m$ contains one effective $\pi$-orbital state, $\left|X_{m}\right\rangle \equiv c_{m}^{\dagger}|0\rangle$. When it is necessary to specify the sublattice $X$, we explicitly write $\left|A_{m}\right\rangle$ and $\left|B_{m}\right\rangle$ for the two atomic sites in a hexagonal lattice. Including also electron spin, $\sigma=\{\uparrow, \downarrow\} \equiv\{+1,-1\}$, the effective one-particle Hilbert space is spanned by states $\left|X_{m} \sigma\right\rangle \equiv c_{m, \sigma}^{\dagger}|0\rangle$.

The structural point group of an ideal hexagonal lattice is the symmetry group $D_{6 h}$-in international crystallographic notation group $6 / \mathrm{mmm}$. It contains 24 group elements which can be expressed in terms of four group generators: identity

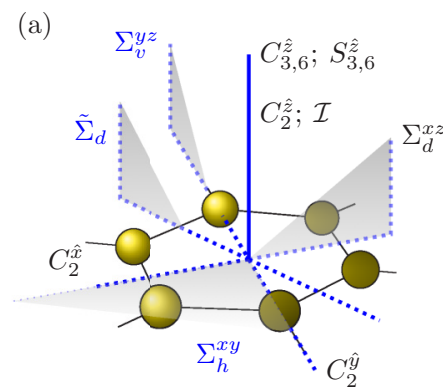

(b)

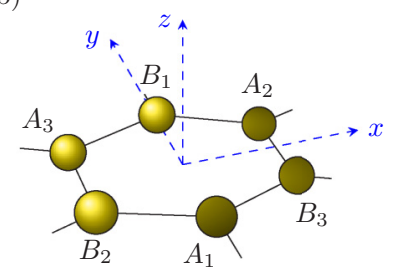

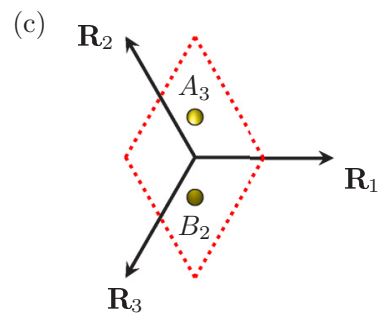

(c)
FIG. 1. Panel (a) shows selected symmetry operations of point group $D_{6 h}$. Combining the point group generators (blue symbols)horizontal $\Sigma_{h}^{x y}$, vertical $\Sigma_{v}^{y z}$, and dihedral $\tilde{\Sigma}_{d}$ reflections, one can built all the remaining group elements (black symbols). Lower panels depict (b) sites' labeling convention and axes orientation, and (c) graphene unit cell together with the Bravais lattice vectors $\mathbf{R}_{\alpha}(\alpha=$ $1,2,3)$.

$E$ and reflections $\Sigma_{h} \equiv \Sigma_{h}^{x y}, \Sigma_{v} \equiv \Sigma_{v}^{y z}, \tilde{\Sigma}_{d}$, for visualization see Fig. 1. Subscripts $h, v$, and $d$ stand for the horizontal ( $x y$ plane), vertical ( $y z$ plane), and dihedral (rotated $x z$ plane) reflections, respectively. When it is convenient to emphasize the reflection planes explicitly, we employ the superscripts $x z$, $y z$, and $x y$. Similarly, to specify the axis determining a spatial rotation we use hat superscripts, such as $\hat{x}, \hat{y}$, and $\hat{z}$. The remaining elements of $D_{6 h}$ are sixfold and threefold rotations, $C_{6}^{\hat{z}}=\tilde{\Sigma}_{d} \circ \Sigma_{v}$ and $C_{3}^{\hat{z}}=C_{6}^{\hat{z}} \circ C_{6}^{\hat{z}}$, the $x z$-dihedral reflection $\Sigma_{d} \equiv \Sigma_{d}^{x z}=C_{3}^{\hat{z}} \circ \tilde{\Sigma}_{d}$, the space inversion $\mathcal{I}=\Sigma_{h} \circ \Sigma_{d} \circ \Sigma_{v}$, the improper rotations $S_{6}^{\hat{z}}=\Sigma_{h} \circ C_{6}^{\hat{z}}, S_{3}^{\hat{z}}=\Sigma_{h} \circ C_{3}^{\hat{z}}$, and the twofold rotations $C_{2}^{\hat{x}}=\Sigma_{h} \circ \Sigma_{d}, C_{2}^{\hat{y}}=\Sigma_{h} \circ \Sigma_{v}$, and $C_{2}^{\hat{z}}=$ $\Sigma_{v} \circ \Sigma_{d}$, see Fig. 1 .

To construct an invariant SOC Hamiltonian, it is necessary to know how the one-particle basis states $\left|X_{m} \sigma\right\rangle$ transform under the active action of $D_{6 h}$ including the time reversal symmetry $\mathcal{T}$. While we are not dealing with the double group irreps it is enough to focus on the action of selected group elements: rotation $\mathcal{R}_{\Phi}^{\hat{z}}$ by an angle $\Phi$, the horizontal, vertical, and dihedral reflections $\Sigma_{h}, \Sigma_{v}$, and $\Sigma_{d}$, respectively, the time reversal $\mathcal{T}$, and for completeness also the space inversion $\mathcal{I}$ and the translation $T_{\vec{a}}$ by a lattice vector $\vec{a}$ :

$$
\begin{aligned}
\left|X_{m} \sigma\right\rangle & \stackrel{\mathcal{R}_{\Phi}}{\longrightarrow} e^{-i \sigma \frac{\Phi}{2}}\left|X_{\mathcal{R}_{\Phi}(m)} \sigma\right\rangle, \\
\left|X_{m} \sigma\right\rangle & \stackrel{\Sigma_{h}^{x y}}{\longrightarrow} i(-1)^{\frac{1+\sigma}{2}}\left|X_{m} \sigma\right\rangle, \\
\left|X_{m} \sigma\right\rangle & \stackrel{\Sigma_{v}^{y z}}{\longrightarrow} i\left|X_{\Sigma_{v}^{y z}(m)}(-\sigma)\right\rangle, \\
\left|X_{m} \sigma\right\rangle & \stackrel{\Sigma_{d}^{x z}}{\longrightarrow}(-1)^{\frac{1+\sigma}{2}}\left|X_{\Sigma_{d}^{x z}(m)}(-\sigma)\right\rangle,
\end{aligned}
$$




$$
\begin{aligned}
& \left|X_{m} \sigma\right\rangle \stackrel{\mathcal{T}}{\rightarrow}(-1)^{\frac{1-\sigma}{2}}\left|X_{m}(-\sigma)\right\rangle, \\
& \left|X_{m} \sigma\right\rangle \stackrel{\mathcal{I}}{\longrightarrow}-\left|X_{\mathcal{I}(m)} \sigma\right\rangle, \\
& \left|X_{m} \sigma\right\rangle \stackrel{T_{\vec{a}}}{\longrightarrow}\left|X_{m+\vec{a}} \sigma\right\rangle .
\end{aligned}
$$

The action of the remaining $D_{6 h}$ elements follow immediately from the relations to the group generators. The action of $\mathcal{T}$ affects only the spin component of $\left|X_{m} \sigma\right\rangle=\left|X_{m}\right\rangle \otimes|\sigma\rangle$ since, by convention, our orbital $\pi$ states $\left|X_{m}\right\rangle$ are real-valued wave functions.

An electron moving in an effective crystal field potential $V$ is affected by SOC interaction that is represented by Hamiltonian,

$$
\hat{H}_{\mathrm{so}}=\frac{\hbar}{4 m_{\mathrm{e}}^{2} \mathrm{c}^{2}}(\nabla V \times \hat{\mathbf{p}}) \cdot \hat{\boldsymbol{s}} .
$$

Here, $m_{\mathrm{e}}$ is the vacuum rest mass of the electron, $\mathrm{c}$ is the speed of light, $\hat{\mathbf{p}}$ stands for the momentum operator, and $\hat{\boldsymbol{s}}=\left(\hat{s}_{x}, \hat{s}_{y}, \hat{s}_{z}\right)$ represents the array of Pauli matrices acting on spin degrees of freedom. In reality, we do not know the crystal field and so $\hat{H}_{\text {so }}$ exactly, but knowing the pool of symmetries preserving $V$, and hence $\hat{H}_{\text {so }}$, we can uniquely detect which matrix elements $\left\langle X_{m} \sigma\left|\hat{H}_{\text {so }}\right| X_{n} \sigma^{\prime}\right\rangle$ are nonzero and thus important. If $\mathcal{S}$ is a system's symmetry-precisely, its unitary representation-then $\mathcal{S} \hat{H}_{\mathrm{so}}=\hat{H}_{\mathrm{so}} \mathcal{S}$ and

$$
\begin{aligned}
\left\langle\mathcal{S}\left[X_{m} \sigma\right]\left|\hat{H}_{\mathrm{so}}\right| \mathcal{S}\left[X_{n} \sigma^{\prime}\right]\right\rangle & =\left\langle\mathcal{S}\left[X_{m} \sigma\right] \mid \mathcal{S}\left[\hat{H}_{\mathrm{so}} X_{n} \sigma^{\prime}\right]\right\rangle \\
& =\left\langle X_{m} \sigma\left|\hat{H}_{\mathrm{so}}\right| X_{n} \sigma^{\prime}\right\rangle
\end{aligned}
$$

for any two one-particle states $\left|X_{m} \sigma\right\rangle$ and $\left|X_{n} \sigma^{\prime}\right\rangle$. In an analogous way, we get for the antiunitary time reversal symmetry, $\mathcal{T} \hat{H}_{\text {so }}=\hat{H}_{\text {so }} \mathcal{T}$, and self-adjoint $\hat{H}_{\text {so }}$

$$
\begin{aligned}
\left\langle\mathcal{T}\left[X_{m} \sigma\right]\left|\hat{H}_{\mathrm{so}}\right| \mathcal{T}\left[X_{n} \sigma^{\prime}\right]\right\rangle & =\left\langle\mathcal{T}\left[X_{m} \sigma\right] \mid \mathcal{T}\left[\hat{H}_{\mathrm{so}} X_{n} \sigma^{\prime}\right]\right\rangle \\
& =\overline{\left\langle X_{m} \sigma\left|\hat{H}_{\mathrm{so}}\right| X_{n} \sigma^{\prime}\right\rangle} \\
& =\left\langle X_{n} \sigma^{\prime}\left|\hat{H}_{\mathrm{so}}\right| X_{m} \sigma\right\rangle .
\end{aligned}
$$

This gives us a practical relation connecting SOC matrix elements with opposite spin projections:

$$
\begin{aligned}
& \left\langle X_{m} \sigma\left|\hat{H}_{\mathrm{so}}\right| X_{n} \sigma^{\prime}\right\rangle \\
& \stackrel{(1 e)}{=}\left\langle(-1)^{-\frac{1-\sigma}{2}} \mathcal{T}\left[X_{m}(-\sigma)\right]\left|\hat{H}_{\mathrm{so}}\right|(-1)^{-\frac{1-\sigma^{\prime}}{2}} \mathcal{T}\left[X_{n}\left(-\sigma^{\prime}\right)\right]\right\rangle \\
& \quad=-(-1)^{\frac{\sigma+\sigma^{\prime}}{2}}\left\langle\mathcal{T}\left[X_{m}(-\sigma)\right]\left|\hat{H}_{\mathrm{so}}\right| \mathcal{T}\left[X_{n}\left(-\sigma^{\prime}\right)\right]\right\rangle \\
& \stackrel{(4)}{=}-(-1)^{\frac{\sigma+\sigma^{\prime}}{2}}\left\langle X_{n}\left(-\sigma^{\prime}\right)\left|\hat{H}_{\mathrm{so}}\right| X_{m}(-\sigma)\right\rangle
\end{aligned}
$$

In practice, we focus only on the on-site, nearest neighbors, and the next-nearest-neighbor SOC mediated hoppings $\left\langle X_{m} \sigma\left|\hat{H}_{\mathrm{so}}\right| X_{n} \sigma^{\prime}\right\rangle$. This is sufficient because the orbital overlaps modulated by $\nabla V$-dominant near the atomic coresdecay rather fast with increasing distance. Therefore we focus on SOC hoppings $\left\langle X_{m} \sigma\left|\hat{H}_{\text {so }}\right| X_{n} \sigma^{\prime}\right\rangle$ inside one particular elementary cell of the hexagonal lattice, see Fig. 1. All other spin-resolved hoppings can be expressed by applying translations, rotations, reflections, or time reversal, see Eqs. (1).

In what follows we show how time reversal symmetry and self-adjointness of $\hat{H}_{\text {so }}$ restrict $\left\langle X_{m} \sigma\left|\hat{H}_{\text {so }}\right| X_{n} \sigma^{\prime}\right\rangle$. Particulary, we argue that the spin-conserving hoppings $\left\langle X_{m} \sigma\left|\hat{H}_{\mathrm{so}}\right| X_{n} \sigma\right\rangle$ are purely imaginary, and the on-site SOC resolved hoppings $\left\langle X_{m} \sigma\left|\hat{H}_{\mathrm{so}}\right| X_{m} \sigma\right\rangle$ and $\left\langle X_{m} \sigma\left|\hat{H}_{\mathrm{so}}\right| X_{m}(-\sigma)\right\rangle$ vanish. First, note that the SOC Hamiltonian $\hat{H}_{\text {so }}$, Eq. (2), can be recast into the form

$$
\hat{H}_{\text {so }}=\hat{\mathcal{L}}_{+} \hat{s}_{-}+\hat{\mathcal{L}}_{-} \hat{s}_{+}+\hat{\mathcal{L}}_{z} \hat{s}_{z},
$$

where $\hat{s}_{ \pm}=\frac{1}{2}\left(\hat{s}_{x} \pm i \hat{s}_{y}\right)$ are spin raising and lowering operators (without $\frac{\hbar}{2}$ ) and $\hat{\mathcal{L}}$ 's act solely on the orbital part of the wave function. It follows from the hermiticity of $\hat{H}_{\text {so }}$ that $\hat{\mathcal{L}}_{-}^{\dagger}=\hat{\mathcal{L}}_{+}$and $\hat{\mathcal{L}}_{z}$ is self-adjoint. Also $\mathcal{L}$ 's transform under the space and time reversal symmetries equally as the standard angular momentum operators. However, for a general crystal field potential $V$ they do not obey the usual $\mathrm{SU}(2)$-commutation relations. Directly from Eq. (6) we have

$$
\left\langle X_{m} \sigma\left|\hat{H}_{\mathrm{so}}\right| X_{n} \sigma\right\rangle=-\left\langle X_{m}(-\sigma)\left|\hat{H}_{\mathrm{so}}\right| X_{n}(-\sigma)\right\rangle \text {. }
$$

On the other side, the time reversal symmetry, Eq. (5), implies

$$
\begin{aligned}
\left\langle X_{m}(-\sigma)\left|\hat{H}_{\mathrm{so}}\right| X_{n}(-\sigma)\right\rangle & \stackrel{(5)}{=}\left\langle X_{n} \sigma\left|\hat{H}_{\mathrm{so}}\right| X_{m} \sigma\right\rangle \\
& =\overline{\left\langle X_{m} \sigma\left|\hat{H}_{\mathrm{so}}\right| X_{n} \sigma\right\rangle .}
\end{aligned}
$$

So comparing this and the above expression, we see that

$$
\left\langle X_{m} \sigma\left|\hat{H}_{\mathrm{so}}\right| X_{n} \sigma\right\rangle
$$

is a purely imaginary SOC matrix element for any two atomic sites mediating a spin-conserving hopping. In the special case $m=n$, the above Eqs. (5) and (7) give

$$
\begin{aligned}
\left\langle X_{m} \sigma\left|\hat{H}_{\mathrm{so}}\right| X_{m} \sigma\right\rangle & \stackrel{(5)}{=}\left\langle X_{m}(-\sigma)\left|\hat{H}_{\mathrm{so}}\right| X_{m}(-\sigma)\right\rangle \\
& \stackrel{(7)}{=}-\left\langle X_{m} \sigma\left|\hat{H}_{\mathrm{so}}\right| X_{m} \sigma\right\rangle,
\end{aligned}
$$

so that we have shown that the on-site spin-conserving term $\left\langle X_{m} \sigma\left|\hat{H}_{\mathrm{so}}\right| X_{m} \sigma\right\rangle$ equals zero for any site $m$. In a similar way we get for its spin-flipping counterpart:

$$
\left\langle X_{m} \sigma\left|\hat{H}_{\mathrm{so}}\right| X_{m}(-\sigma)\right\rangle \stackrel{(5)}{=}-\left\langle X_{m} \sigma\left|\hat{H}_{\mathrm{so}}\right| X_{m}(-\sigma)\right\rangle,
$$

so the on-site spin-flipping matrix element $\left\langle X_{m} \sigma\left|\hat{H}_{\mathrm{so}}\right|\right.$ $\left.X_{m}(-\sigma)\right\rangle$ is zero for any lattice site $m$. Therefore what matters are the nearest- and next-nearest-neighbor SOC mediated matrix elements, which we will examine in the forthcoming sections.

\section{TRANSLATIONAL INVARIANT SYSTEMS}

\section{A. Pristine graphene SOC Hamiltonian}

The spin-orbit coupling Hamiltonian based on $\pi$ states that is translational invariant and possesses the full point group symmetry $D_{6 h}$ of the pristine graphene allows only one, the so called intrinsic, SOC hopping $\lambda_{\mathrm{I}}$. This was first discussed by McClure and Yafet [81] when analyzing the $g$ factor in a "graphite single crystal." Later Kane and Mele [32] revisited this point when predicting the quantum spin Hall effect in graphene. The magnitude of $\lambda_{I}$ was found in the work of Gmitra et al. [9], who showed that $\lambda_{\mathrm{I}}$ is too weak-about $12 \mu \mathrm{eV}$ - to induce an experimentally detectable transition into the quantum spin Hall phase. Furthermore, Gmitra et al. [9] found that $\lambda_{\mathrm{I}}$ is due to the coupling of $p_{z}$ and $d$ orbitals. This was supported by multiorbital tight-binding calculations; Konschuh et al. [75] showed that the intrinsic SOC hopping 
$\lambda_{\mathrm{I}}$ is significantly affected by the admixture of $3 d_{x z} \pm i 3 d_{y z}$ orbitals, the fact anticipated already by Slonczewski [72].

The effective tight-binding Hamiltonian mediating the SOC interaction among $\pi$ states in graphene-or any planar hexagonal system with one $\pi$-orbital per site-reads

$$
\mathcal{H}_{D_{6 h}}=\frac{i \lambda_{\mathrm{I}}}{3 \sqrt{3}} \sum_{\sigma} \sum_{\langle\langle m, n\rangle\rangle} v_{m, n}\left[\hat{s}_{z}\right]_{\sigma \sigma}\left|X_{m} \sigma\right\rangle\left\langle X_{n} \sigma\right| .
$$

The Hamiltonian $\mathcal{H}_{D_{6 h}}$ couples next-nearest neighbors (summation over $\langle\langle m, n\rangle\rangle)$ and allows only spin-conserving hoppings. Therefore, in accordance with Eq. (9), the underlying coupling constant is purely imaginary. Using the configuration shown at Fig. 1, the coupling $i \lambda_{\mathrm{I}}$ can be defined as

$$
\frac{i \lambda_{\mathrm{I}}}{3 \sqrt{3}}=\left\langle A_{3} \uparrow\left|\hat{H}_{\text {so }}\right| A_{2} \uparrow\right\rangle \stackrel{(7)}{=}-\left\langle A_{3} \downarrow\left|\hat{H}_{\text {so }}\right| A_{2} \downarrow\right\rangle .
$$

The numerical factor $1 / 3 \sqrt{3}$ is a matter of convention; adding it here, the low-energy expansion of the Bloch transform of $\mathcal{H}_{D_{6 h}}$ becomes simpler. In the above formula and also below, we identify a lattice site $m$ with a $\pi$ state $\left|X_{m}\right\rangle$ residing on it. Since each site hosts one $\pi$-orbital state, this assignment is unique. Moreover, since $\left|A_{2} \uparrow\right\rangle=\Sigma_{d}^{x z}\left|B_{3} \downarrow\right\rangle$, and $\left|A_{3} \uparrow\right\rangle=$ $\Sigma_{d}^{x z}\left|B_{2} \downarrow\right\rangle$, see Fig. 1, we can write

$$
\begin{gathered}
\frac{i \lambda_{\mathrm{I}}}{3 \sqrt{3}}=\left\langle A_{3} \uparrow\left|\hat{H}_{\mathrm{so}}\right| A_{2} \uparrow\right\rangle \stackrel{(1 d)}{=}\left\langle\Sigma_{d}^{x z}\left[B_{2} \downarrow\right]\left|\hat{H}_{\mathrm{so}}\right| \Sigma_{d}^{x z}\left[B_{3} \downarrow\right]\right\rangle \\
\stackrel{(3)}{=}\left\langle B_{2} \downarrow\left|\hat{H}_{\mathrm{so}}\right| B_{3} \downarrow\right\rangle \stackrel{(7)}{=}-\left\langle B_{2} \uparrow\left|\hat{H}_{\mathrm{so}}\right| B_{3} \uparrow\right\rangle .
\end{gathered}
$$

All the sublattice and spin related sign factors are captured in the prefactor term $v_{m, n}\left[\hat{s}_{z}\right]_{\sigma \sigma}$, i.e.,

$$
\left\langle X_{m} \sigma\left|\hat{H}_{\mathrm{so}}\right| X_{n} \sigma\right\rangle=v_{m, n}\left[\hat{s}_{z}\right]_{\sigma \sigma} \frac{i \lambda_{\mathrm{I}}}{3 \sqrt{3}} .
$$

There, $v_{m, n}=+1(-1)$, if the next-nearest-neighbor hopping $n \rightarrow m$ via a common neighbor on the opposite sublattice is counter clockwise (clockwise), e.g., for $A_{2} \rightarrow\left(B_{1}\right) \rightarrow A_{3}$, $v_{A_{3} A_{2}}=+1$, while for $B_{3} \rightarrow\left(A_{1}\right) \rightarrow B_{2}, v_{B_{2} B_{3}}=-1$, see Fig. 1. The dependence on spin $\sigma$ is governed by $\left[\hat{s}_{z}\right]_{\sigma \sigma}$; as defined $\left[\hat{s}_{z}\right]_{ \pm \pm}= \pm 1$.

To see the effect of the intrinsic SOC on the band structure we transform $\mathcal{H}_{D_{6 h}}$, Eq. (12), from the local atomic into the Bloch basis, $\left|X_{m} \sigma\right\rangle \mapsto\left|X_{\mathbf{q}} \sigma\right\rangle$ :

$$
\left|X_{\mathbf{q}} \sigma\right\rangle=\frac{1}{\sqrt{N_{1} N_{2}}} \sum_{\mathbf{R}_{m}} e^{i \mathbf{q} \cdot \mathbf{R}_{m}}\left|X_{m} \sigma\right\rangle .
$$

Here, $X=\{A, B\}$ and $\sigma=\{\uparrow, \downarrow\}$, dependent on the sublattice and spin degrees of freedom, respectively, $\mathbf{q}$ is the quasimomentum measured from the center of the hexagonal Brillouin zone ( $\Gamma$ point), $N_{1} N_{2}$ is the number of graphene unit cells in the sample, and $\mathbf{R}_{m}$ is the lattice vector of the $m$ th cell that hosts the orbital $\left|X_{m} \sigma\right\rangle$. Inserting the above unitary transformation into Eq. (12), we transform $\mathcal{H}_{D_{6 h}}$ to the Bloch form, $\mathcal{H}_{D_{6 h}}=\sum_{\mathbf{q}} \mathcal{H}_{D_{6 h}}(\mathbf{q})$, where

$$
\mathcal{H}_{D_{6 h}}(\mathbf{q})=\lambda_{\mathrm{I}} f_{\mathrm{I}}(\mathbf{q}) \sum_{X, \sigma}\left[\hat{\sigma}_{z}\right]_{X X}\left[\hat{s}_{z}\right]_{\sigma \sigma}\left|X_{\mathbf{q}} \sigma\right\rangle\left\langle X_{\mathbf{q}} \sigma\right| .
$$

Here, the Pauli matrix $\hat{\sigma}_{z}$ acts in the space of sublattices$\left[\hat{\sigma}_{z}\right]_{A A}=1=-\left[\hat{\sigma}_{z}\right]_{B B}$, and $\left[\hat{\sigma}_{z}\right]_{A B}=0=\left[\hat{\sigma}_{z}\right]_{B A}$. The intrinsic structural function $f_{\mathrm{I}}(\mathbf{q})$ reads,

$$
f_{\mathrm{I}}(\mathbf{q})=-\frac{2}{3 \sqrt{3}}\left(\sin \mathbf{q} \cdot \mathbf{R}_{1}+\sin \mathbf{q} \cdot \mathbf{R}_{2}+\sin \mathbf{q} \cdot \mathbf{R}_{3}\right) .
$$

The lattice vectors $\mathbf{R}_{\alpha}(\alpha=1,2,3)$ can be compactly expressed in terms of the Levi-Civita antisymmetric $\epsilon$ symbol and the position vectors of the lattice sites $A_{1}, A_{2}$, and $A_{3}$ as displayed at Fig. 1. Particularly,

$\mathbf{R}_{\alpha}=\frac{1}{2} \epsilon_{\alpha \beta \gamma} \overrightarrow{A_{\gamma} A_{\beta}}=a_{\mathrm{L}}\left(\cos \frac{2 \pi(\alpha-1)}{3}, \sin \frac{2 \pi(\alpha-1)}{3}\right)$,

where $a_{\mathrm{L}}$ is the lattice constant; in the case of graphene $a_{\mathrm{L}}=$ $2.46 \AA$.

On the orbital level the electronic band structure of graphene $\pi$ orbitals is well described by the standard nearestneighbor Hamiltonian,

$$
\mathcal{H}_{\text {orb }}=-t \sum_{\sigma} \sum_{\langle m, n\rangle}\left|X_{m} \sigma\right\rangle\left\langle X_{n} \sigma\right|,
$$

with $t=2.6 \mathrm{eV}$. Transforming it to the Bloch form, we arrive at $\mathcal{H}_{\text {orb }}=\sum_{\mathbf{q}} \mathcal{H}_{\text {orb }}(\mathbf{q})$, where

$$
\mathcal{H}_{\text {orb }}(\mathbf{q})=-t \sum_{\sigma} f_{\text {orb }}(\mathbf{q})\left[\hat{s}_{0}\right]_{\sigma \sigma}\left|A_{\mathbf{q}} \sigma\right\rangle\left\langle B_{\mathbf{q}} \sigma\right|+\text { H.c. },
$$

and the orbital structural function is given by

$$
f_{\text {orb }}(\mathbf{q})=\left(1+\mathrm{e}^{i \mathbf{q} \cdot \mathbf{R}_{2}}+\mathrm{e}^{-i \mathbf{q} \cdot \mathbf{R}_{3}}\right)
$$

$\hat{s}_{0}$ is the identity matrix in spin space.

In what follows, we focus on the low-energy physics near the Dirac points,

$$
\pm \mathbf{K}= \pm \frac{4 \pi}{3 a_{\mathrm{L}}}(1,0)
$$

i.e., we substitute for $\mathbf{q}= \pm \mathbf{K}+\mathbf{k}$ and expand the relevant $\mathbf{q}$-dependent quantities in $\mathbf{k}$ keeping the first nonzero term. For the above defined structural functions, we particularly get

$$
f_{\mathrm{I}}( \pm \mathbf{K}+\mathbf{k}) \simeq \pm 1
$$

and

$$
f_{\text {orb }}( \pm \mathbf{K}+\mathbf{k}) \simeq \frac{\sqrt{3} a_{\mathrm{L}}}{2}\left(\mp k_{x}-i k_{y}\right) .
$$

Fixing the order $\left\{\left|A_{\mathbf{q}} \uparrow\right\rangle,\left|A_{\mathbf{q}} \downarrow\right\rangle,\left|B_{\mathbf{q}} \uparrow\right\rangle,\left|B_{\mathbf{q}} \downarrow\right\rangle\right\}$ of the Bloch basis, we arrive at the effective low-energy Hamiltonian in the form

$$
\mathcal{H}_{\mathrm{eff}}(\tau \mathbf{K}+\mathbf{k})=\hbar v_{F}\left(\tau k_{x} \hat{\sigma}_{x}-k_{y} \hat{\sigma}_{y}\right) \hat{s}_{0}+\tau \lambda_{\mathrm{I}} \hat{\sigma}_{z} \hat{s}_{z} .
$$

Here, $\tau \mathbf{K}= \pm \mathbf{K}$ is the shorthand for the Dirac valleys, $\hat{\sigma}_{x(y)}$ are Pauli matrices in the sublattice space, and $v_{F}=\sqrt{3} a_{\mathrm{L}} t / 2 \hbar$ stands for the Fermi velocity; for example, for graphene $v_{F} \approx$ $10^{6} \mathrm{~m} / \mathrm{s}$.

From the above Bloch representation, we see that $\hat{\sigma}_{0} \hat{s}_{z}$ commutes with $\mathcal{H}_{\text {eff }}$, and hence its eigenstates can be labeled by the spin $\uparrow$ and $\downarrow$ projections along the $z$ spin quantization axis independently of $\mathbf{k}$. The eigenspectrum of $\mathcal{H}_{\text {eff }}$, dependent on the quasimomentum $\mathbf{k}$, band index $n=+/-=$ conduction/valence, and spin $\sigma=\{\uparrow, \downarrow\}$, reads

$$
\varepsilon_{n, \sigma}(\tau \mathbf{K}+\mathbf{k})=n \sqrt{\lambda_{\mathrm{I}}^{2}+\hbar^{2} v_{F}^{2}\left(k_{x}^{2}+k_{y}^{2}\right)} .
$$




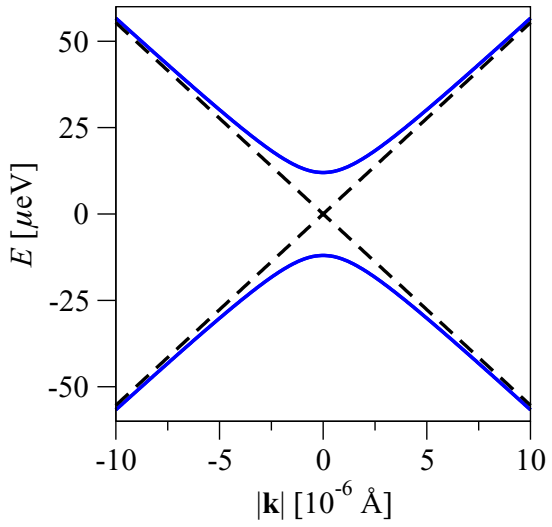

FIG. 2. Electronic band structure of the typical graphenelike system in the vicinity of the Dirac point $(|\mathbf{k}|=0)$ with (solid line) and without (dashed line) the intrinsic ( $D_{6 h}$ invariant) SOC Hamiltonian. The spectral gap of $2 \lambda_{\mathrm{I}}$ and the parabolic shape near $|\mathbf{k}|=0$ are typical imprints of the intrinsic SOC which does not spin-split the bands.

The corresponding four eigenstates get grouped into pairs, each pair comprising states with the opposite spins, e.g., directly at the $\tau \mathbf{K}$ points, we have two pairs $\left\{\left|A_{\tau \mathbf{K}} \uparrow\right\rangle,\left|B_{\tau \mathbf{K}} \downarrow\right\rangle\right\}$ and $\left\{\left|A_{\tau \mathbf{K}} \downarrow\right\rangle,\left|B_{\tau \mathbf{K}} \uparrow\right\rangle\right\}$, that are split in energy by the intrinsic SOC; spin-orbit interaction opens a spectral gap at the Dirac points. In the case of graphene, the intrinsic gap equals [9] $2 \lambda_{\mathrm{I}} \simeq 24 \mu \mathrm{eV}$. The spectral effects of the intrinsic SOC that are imprinted on the band structure are shown at Fig. 2.

\section{B. No-go SOC matrix elements-lethal symmetries}

In what follows, we shortly summarize no-go arguments showing explicitly how certain SOC mediated matrix elements become inhibited by specific structural symmetries. This will on one hand prove why for pristine graphene only the spin-conserving next-nearest-neighbor coupling $\lambda_{\mathrm{I}}$ is allowed. On the other hand, by seeing the absence of a particular no-go symmetry in the symmetry group of a reduced hexagonal structure we can infer which additional coupling is allowed in the corresponding effective SOC Hamiltonian. We will profit from this insight in the forthcoming sections.

\section{Inhibition of all spin-flip SOCs-horizontal reflection}

Applying horizontal reflection $\Sigma_{h}^{x y}$ to a general spin-flip matrix element $\left\langle X_{m} \sigma\left|\hat{H}_{\mathrm{so}}\right| X_{n}(-\sigma)\right\rangle$ between two $\pi$ states localized on arbitrary lattice sites $m$ and $n$, we get in accordance with Eq. (1b),

$$
\begin{aligned}
& \left\langle X_{m} \sigma\left|\hat{H}_{\mathrm{so}}\right| X_{n}(-\sigma)\right\rangle \\
& =\left\langle i(-1)^{\frac{1-\sigma}{2}} \Sigma_{h}^{x y}\left[X_{m} \sigma\right]\left|\hat{H}_{\mathrm{so}}\right| i(-1)^{\frac{1+\sigma}{2}} \Sigma_{h}^{x y}\left[X_{n}(-\sigma)\right]\right\rangle \\
& =-\left\langle\Sigma_{h}^{x y}\left[X_{m} \sigma\right]\left|\hat{H}_{\mathrm{so}}\right| \Sigma_{h}^{x y}\left[X_{n}(-\sigma)\right]\right\rangle \\
& \stackrel{(3)}{=}-\left\langle X_{m} \sigma\left|\hat{H}_{\mathrm{so}}\right| X_{n}(-\sigma)\right\rangle,
\end{aligned}
$$

what implies that $\left\langle X_{m} \sigma\left|\hat{H}_{\text {so }}\right| X_{n}(-\sigma)\right\rangle=0$. Hence we showed that the presence of $\Sigma_{h}^{x y}$ in the reduced point group inhibits any spin-flip terms in the effective SOC Hamiltonian. If $\Sigma_{h}^{x y}$ would not be present, then we would have a weaker result as discussed below.

\section{Inhibition of the nearest-neighbor spin-flip}

\section{SOCs-space inversion, lattice translation, and time reversal}

For concreteness, let us focus on the SOC matrix element $\left\langle A_{2} \sigma\left|\hat{H}_{\text {so }}\right| B_{3}(-\sigma)\right\rangle$; see Fig. 1. Employing consecutively space inversion $\mathcal{I}$, Eq. (1f), unitarity, Eq. (3), and translation by the lattice vector $\vec{a}=\overrightarrow{A_{3} A_{2}}=\overrightarrow{B_{2} B_{3}}$, Eq. (1g), we get

$$
\begin{aligned}
\left\langle A_{2} \sigma\left|\hat{H}_{\mathrm{so}}\right| B_{3}(-\sigma)\right\rangle & =\left\langle-\mathcal{I}\left[B_{2} \sigma\right]\left|\hat{H}_{\mathrm{so}}\right|-\mathcal{I}\left[A_{3}(-\sigma)\right]\right\rangle \\
& =\left\langle\mathcal{I}\left[B_{2} \sigma\right]\left|\hat{H}_{\mathrm{so}}\right| \mathcal{I}\left[A_{3}(-\sigma)\right]\right\rangle \\
& \stackrel{(3)}{=}\left\langle B_{2} \sigma\left|\hat{H}_{\mathrm{so}}\right| A_{3}(-\sigma)\right\rangle \\
& =\left\langle T_{\vec{a}}\left[B_{3} \sigma\right]\left|\hat{H}_{\mathrm{so}}\right| T_{\vec{a}}\left[A_{2}(-\sigma)\right]\right\rangle \\
& \stackrel{(3)}{=}\left\langle B_{3} \sigma\left|\hat{H}_{\mathrm{so}}\right| A_{2}(-\sigma)\right\rangle .
\end{aligned}
$$

To proceed further, we use the time reversal symmetry, Eq. (5),

$$
\left\langle B_{3} \sigma\left|\hat{H}_{\text {so }}\right| A_{2}(-\sigma)\right\rangle \stackrel{(5)}{=}-\left\langle A_{2} \sigma\left|\hat{H}_{\text {so }}\right| B_{3}(-\sigma)\right\rangle .
$$

Combining Eqs. (28a) and (28b), we immediately see that the nearest-neighbor SOC mediated spin-flip hopping $\left\langle A_{2} \sigma\left|\hat{H}_{\text {so }}\right| B_{3}(-\sigma)\right\rangle=0$. Repeating the same for the remaining neighboring lattice sites at Fig. 1 we inhibit-by the space inversion $\mathcal{I}$, lattice translation $T_{\vec{a}}$ and time reversal $\mathcal{T}$-all other nearest-neighbor spin-flip terms in the effective SOC Hamiltonian.

\section{Inhibition of the nearest-neighbor spin-conserving SOCs-vertical reflection and lattice translation}

By similar reasoning as above, we can show that the SOC matrix element $\left\langle A_{2} \sigma\left|\hat{H}_{\text {so }}\right| B_{3} \sigma\right\rangle$ is zero whenever lattice translation $T_{\vec{a}}$ and vertical reflection $\Sigma_{v}^{y z}$ are present. Translation by the lattice vector $\vec{a}=\overrightarrow{A_{2} A_{3}}=\overrightarrow{B_{3} B_{2}}$ implies

$$
\begin{aligned}
\left\langle A_{2} \sigma\left|\hat{H}_{\mathrm{so}}\right| B_{3} \sigma\right\rangle & =\left\langle T_{\vec{a}}\left[A_{3} \sigma\right]\left|\hat{H}_{\mathrm{so}}\right| T_{\vec{a}}\left[B_{2} \sigma\right]\right\rangle \\
& \stackrel{(3)}{=}\left\langle A_{3} \sigma\left|\hat{H}_{\mathrm{so}}\right| B_{2} \sigma\right\rangle .
\end{aligned}
$$

Moreover, using the vertical reflection, Eq. (1c), we have $\left|A_{3} \sigma\right\rangle=-i \Sigma_{v}^{y z}\left|A_{2}(-\sigma)\right\rangle$ and $\left|B_{2} \sigma\right\rangle=-i \Sigma_{v}^{y z}\left|B_{3}(-\sigma)\right\rangle$ and therefore, by unitarity, Eq. (3), we arrive at

$$
\left\langle A_{3} \sigma\left|\hat{H}_{\mathrm{so}}\right| B_{2} \sigma\right\rangle=\left\langle A_{2}(-\sigma)\left|\hat{H}_{\mathrm{so}}\right| B_{3}(-\sigma)\right\rangle \text {. }
$$

So the last two equations together with Eq. (7) imply

$$
\begin{aligned}
\left\langle A_{2} \sigma\left|\hat{H}_{\mathrm{so}}\right| B_{3} \sigma\right\rangle & =\left\langle A_{2}(-\sigma)\left|\hat{H}_{\mathrm{so}}\right| B_{3}(-\sigma)\right\rangle \\
& \stackrel{(7)}{=}-\left\langle A_{2} \sigma\left|\hat{H}_{\mathrm{so}}\right| B_{3} \sigma\right\rangle,
\end{aligned}
$$

which means that the nearest-neighbor spin-conserving hopping $\left\langle A_{2} \sigma\left|\hat{H}_{\text {so }}\right| B_{3} \sigma\right\rangle$ is zero. Repeating the same argumentation for the other neighboring sites we eliminate-by lattice translation $T_{\vec{a}}$ and vertical reflection $\Sigma_{v}^{y z}$-all remaining nearest-neighbor spin-conserving SOC terms.

The no-go arguments based on the horizontal and vertical reflections $\Sigma_{h}^{x y}, \Sigma_{v}^{y z} \in D_{6 h}$, see Eqs. (27) and (29), explain straightforwardly why the translationally invariant SOC Hamiltonian $\mathcal{H}_{D_{6 h}}$ of pristine graphene, Eq. (12), allows only 


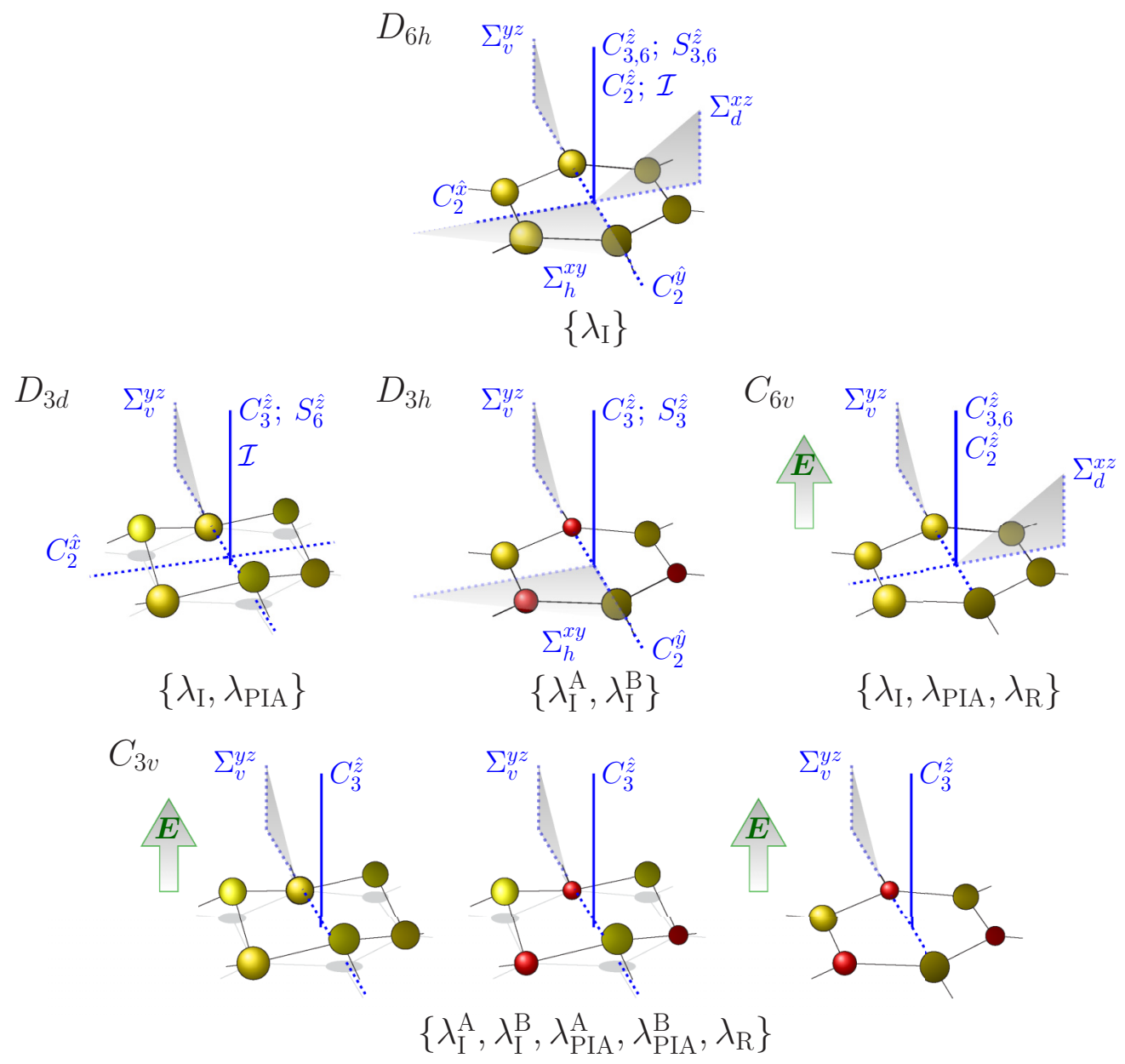

FIG. 3. Point group $D_{6 h}$ of pristine graphene (top), its maximal subgroups $D_{3 d}, D_{3 h}$, and $C_{6 v}$ (second row), and the corresponding symmetry operations (blue symbols). Subgroups $D_{3 d}, D_{3 h}$, and $C_{6 v}$ are represented, for instance, by graphene mini-ripple, planar boron-nitride and graphene exposed to a transverse external electric field, respectively. They share the common subgroup $C_{3 v}$ (third row) what is, for example, the symmetry group (left to right) of graphene mini-ripple in a transverse external electric field, mini-rippled boron-nitride, and boron-nitride in a transverse external electric field. The successive reduction of the point group symmetry (top to bottom) enhances the number of symmetry-allowed SOC parameters, those are summarized in brackets.

the next-nearest-neighbor spin-conserving hoppings. Pristine graphene is an example of a hexagonal system with the highest structural group symmetry. The topic for the next sections are hexagonal systems with lower symmetries-subgroups of the point group $D_{6 h}$. We will start with the maximal structural subgroups $D_{3 d}, D_{3 h}$, and $C_{6 v}$-and explore step-by-step the symmetry allowed spin-orbit couplings.

\section{Subgroups of $D_{6 h}$-categorization of emergent SOCs}

Any periodic modification of the pristine hexagonal symmetry reduces the unit cell point group symmetry $D_{6 h}$ to one of its subgroups and is manifested by the emergence of new SOC mediated hoppings. The aim of this section is to show a bottom line enabling their classification and categorization.

The minimal structural modifications we will discuss here are (1) rippling, (2) sublattice asymmetry, and (3) transverse electric field or substrate and their mutual combinations, see Fig. 3. We call here a structural modification of the full hexagonal lattice minimal, if the reduced point subgroup of $D_{6 h}$ experiences minimal modifications in terms of the number of group elements. Such subgroups are usually called maximal subgroups. In the case of $D_{6 h}$, there are in total five maximal subgroups [70]. Each of them has 12 group elements-group order 12-which is half of the order of the original point group $D_{6 h}$. Three subgroups- $D_{3 d}, D_{3 h}$, and $C_{6 v}$-will be relevant in the present context, while the subgroups $D_{6}$ and $C_{6 h}$ are irrelevant for us. To be specific, (1) rippling reduces $D_{6 h} \rightarrow D_{3 d}$ what constitutes the point group of graphene miniripple, graphane [82], silicene, and "germanene" [83,84], etc.; (2) sublattice inversion asymmetry reduces $D_{6 h} \rightarrow D_{3 h}$ what is the point group of the planar boron nitride, aluminum nitride, or any other planar system with two nonequivalent interpenetrating triangular lattices $A$ and $B$; (3) transverse electric field reduces $D_{6 h} \rightarrow C_{6 v}$ what represents the point group of pristine graphene in an external field or graphene deposited on a substrate that is not breaking the sublattice symmetry. For visualization, summary, and mutual comparison see Fig. 3 and Table I.

It is worth to emphasize that an intersection of any two of $D_{3 d}, D_{3 h}$, and $C_{6 v}$ is isomorphic [70] to the smaller non-Abelian subgroup $C_{3 v} \subset D_{6 h}$ with group order 6 . This 
TABLE I. Point group $D_{6 h}$ and its maximal subgroups $-D_{3 d}, D_{3 h}, C_{6 v}$-including also their common intersection-the point group $C_{3 v}$. For the visualization see Fig. 3 . We shortened the notation in terms of the previous definitions: symbol $2 C_{6}^{\hat{z}}$ means two sixfold rotations along the $z$ axis, namely, $\mathcal{R}_{ \pm \pi / 3}^{\hat{z}}$, symbol $3 C_{2}^{\prime}$ stands for three twofold rotations along the axis $x, \mathcal{R}_{\pi / 3}^{\hat{z}} x$, and $\mathcal{R}_{2 \pi / 3}^{\hat{z}} x$, respectively, and similarly $3 C_{2}^{\prime \prime}$ stands for three twofold rotations along the $y, \mathcal{R}_{\pi / 3}^{\hat{z}} y$, and $\mathcal{R}_{2 \pi / 3}^{\hat{z}} y$ axis, respectively. By the same logic, $3 \Sigma_{v}$ stands for three mirror reflections in $y z, \mathcal{R}_{\pi / 3}^{\hat{z}} y z$, and $\mathcal{R}_{2 \pi / 3}^{\hat{z}} y z$ planes, respectively, and so on. If the given set of operations is present/absent in the particular subgroup of $D_{6 h}$, we employ the marker $\checkmark /-$.

\begin{tabular}{lccccccccccc}
\hline \hline Group/Operation & $E$ & $2 C_{6}^{\hat{z}}$ & $2 C_{3}^{\hat{z}}$ & $C_{2}^{\hat{z}}$ & $3 C_{2}^{\prime}$ & $3 C_{2}^{\prime \prime}$ & $\mathcal{I}$ & $\Sigma_{h}$ & $3 \Sigma_{v}$ & $3 \Sigma_{d}$ & $2 S_{3}^{\hat{z}}$ \\
\hline$D_{6 h}$ & $\checkmark$ & $\checkmark$ & $\checkmark$ & $\checkmark$ & $\checkmark$ & $\checkmark$ & $\checkmark$ & $\checkmark$ & $\checkmark$ & $\checkmark$ & $\checkmark$ \\
$D_{3 d}$ & $\checkmark$ & - & $\checkmark$ & - & $\checkmark$ & - & $\checkmark$ & - & $\checkmark$ & - & - \\
$D_{3 h}$ & $\checkmark$ & - & $\checkmark$ & - & - & $\checkmark$ & - & $\checkmark$ & $\checkmark$ & - & $\checkmark$ \\
$C_{6 v}$ & $\checkmark$ & $\checkmark$ & $\checkmark$ & $\checkmark$ & - & - & - & - & $\checkmark$ & $\checkmark$ & - \\
$C_{3 v}$ & $\checkmark$ & - & $\checkmark$ & - & - & - & - & - & $\checkmark$ & - & - \\
\hline \hline
\end{tabular}

means that an arbitrary combination of two minimal structural modifications leads to the same effective SOC Hamiltonian, which possesses global $C_{3 v}$ invariance. For concreteness, graphene miniripple (or graphane, silicene, and germanene) in a transverse electric field- $D_{3 d} \cap C_{6 v}$-is from the effective SOC point of view equivalent to a minirippled boron nitride without the field or free standing graphone [85]- $D_{3 d} \cap D_{3 h}$.

With respect to the structural minimality the point groups $D_{3 d}, D_{3 h}$, and $C_{6 v}$ can be considered as equivalent since they are all maximal subgroups of $D_{6 h}$. Despite of that minimal subgroup similarity, $D_{3 d}, D_{3 h}$, and $C_{6 v}$ are different since they result in different $S O C$ phenomena.

\section{D ${ }_{3 d}$ case: $\lambda_{\mathrm{I}}$ and $\lambda_{\mathrm{PIA}}$ couplings}

Rippled structures such as graphane, silicene, and graphene miniripple-point group $D_{3 d}$-remain invariant under the space inversion $\mathcal{I}$ and time reversal $\mathcal{T}$, and hence SOC can not cause band spin splittings. The reasoning is finger counting [2]; for any band index $n$, we have

$$
\varepsilon_{n, \sigma}(\mathbf{k}) \stackrel{\mathcal{I}}{=} \varepsilon_{n,-\sigma}(-\mathbf{k}) \stackrel{\mathcal{I}}{=} \varepsilon_{n,-\sigma}(\mathbf{k}) .
$$

Space inversion $\mathcal{I}$ and vertical reflection $\Sigma_{v}^{y z}$ belong to $D_{3 d}$, but the horizontal reflection $\Sigma_{h}^{x y}$ does not. Then, according to the no-go arguments presented in Sec. III B, the $D_{3 d}$ symmetric and time reversal invariant SOC Hamiltonian based on $\pi$ orbitals allows only next-nearest-neighbor hoppings. The nearest-neighbor SOCs are inhibited-the spin-conserving ones by $\Sigma_{v}^{y z}$ and spin-flipping by $\mathcal{I}$. Because $\mathcal{I}$ interchanges sublattices, $\left|A_{i} \sigma\right\rangle=-\mathcal{I}\left|B_{i} \sigma\right\rangle$, see Fig. 1 and Eq. (1f), the next-nearest hoppings should not be sublattice resolved. Indeed,

$$
\left\langle A_{i} \sigma\left|\hat{H}_{\mathrm{so}}\right| A_{j} \sigma^{\prime}\right\rangle \stackrel{(3)}{=}\left\langle B_{i} \sigma\left|\hat{H}_{\mathrm{so}}\right| B_{j} \sigma^{\prime}\right\rangle .
$$

Similarly, $\mathcal{T}$ interchanges the spin components,

$$
\begin{aligned}
& \left\langle X_{m} \sigma\left|\hat{H}_{\mathrm{so}}\right| X_{n} \sigma^{\prime}\right\rangle \\
& \stackrel{(5)}{=}-(-1)^{\frac{\sigma+\sigma^{\prime}}{2}}\left\langle X_{n}\left(-\sigma^{\prime}\right)\left|\hat{H}_{\mathrm{so}}\right| X_{m}(-\sigma)\right\rangle,
\end{aligned}
$$

and hence there is only one purely imaginary spin-conserving hopping, say, defined for $\sigma^{\prime}=\sigma=\uparrow$, and one spin-flipping hopping defined for $\sigma^{\prime}=-\sigma=\downarrow$, respectively.

It is now a convention-by analogy with the plain graphene-to call the spin-conserving next-nearest-neighbor SOC matrix element intrinsic. Hence also in the $D_{3 d}$ case we adopt the term intrinsic SOC. We define intrinsic $i \lambda_{\mathrm{I}}$ by the same prescription as already given by Eq. (13):

$$
\frac{i \lambda_{\mathrm{I}}}{3 \sqrt{3}}=\left\langle A_{3} \uparrow\left|\hat{H}_{\mathrm{so}}\right| A_{2} \uparrow\right\rangle=\left\langle B_{2} \downarrow\left|\hat{H}_{\mathrm{so}}\right| B_{3} \downarrow\right\rangle .
$$

The related sublattice-spin sign factors are governed by the prefactor $v_{m, n}\left[\hat{s}_{z}\right]_{\sigma \sigma}$ as discussed above.

There is no terminological consensus on how to call the spin-flipping next-nearest-neighbor SOC matrix element. Such a term already emerged in bilayer graphene [86], but that time its group symmetry origin was not discussed. Later, when studying SOC effects in semi-hydrogenated graphene (graphone) the acronym PIA - a shorthand for the "pseudospin inversion asymmetry" was proposed [34]. In that case, the pseudospin was explicitly broken by the hydrogenation of one sublattice resulting in the $C_{3 v}$ invariant structure. Unfortunately, the pseudospin asymmetry is not supported by the point group $D_{3 d}$ which contains the space inversion $\mathcal{I}$. So the former PIA acronym is not fully appropriate in $D_{3 d}$ case. Alternatively, authors of Ref. [77] used the term "intrinsic Rashba SOC." This is also inappropriate, since normally the Rashba [87] SOC causes band splittings and this is also not the case in $D_{3 d}$ invariant systems.

The emergence of the spin-flipping next-nearest-neighbor SOC is related to the absence of the horizontal reflection $\Sigma_{h}^{x y}$ in the underlying point group (see also other cases discussed below). Since the horizontal plane is a principal mirror plane of the structure we can call it "principal plane mirror asymmetry" induced SOC, preserving the subscript PIA (by explicitly breaking with abbreviation rule). Thus the PIA spin-orbit coupling $\lambda_{\text {PIA }}$ can be defined as

$$
\frac{2}{3} \lambda_{\text {PIA }} \equiv\left\langle A_{3} \uparrow\left|\hat{H}_{\text {so }}\right| A_{2} \downarrow\right\rangle \stackrel{(5)}{=}-\left\langle A_{2} \uparrow\left|\hat{H}_{\text {so }}\right| A_{3} \downarrow\right\rangle .
$$

Again, the numerical prefactor $2 / 3$ is a matter of convenience. Employing the vertical reflection $\Sigma_{v}^{y z} \in D_{3 d}$ we show that $\lambda_{\text {PIA }}$ is purely real

$$
\begin{aligned}
\left\langle A_{3} \uparrow\left|\hat{H}_{\mathrm{so}}\right| A_{2} \downarrow\right\rangle & \stackrel{(1 c)}{=}\left\langle-i \Sigma_{v}^{y z}\left[A_{2} \downarrow\right]\left|\hat{H}_{\mathrm{so}}\right|-i \Sigma_{v}^{y z}\left[A_{3} \uparrow\right]\right\rangle \\
& \stackrel{(3)}{=}\left\langle A_{2} \downarrow\left|\hat{H}_{\mathrm{so}}\right| A_{3} \uparrow\right\rangle=\overline{\left\langle A_{3} \uparrow\left|\hat{H}_{\mathrm{so}}\right| A_{2} \downarrow\right\rangle}
\end{aligned}
$$


As a consequence of the last two equations, we have the practical identity:

$$
\frac{2}{3} \lambda_{\text {PIA }}=\left\langle A_{3} \uparrow\left|\hat{H}_{\text {so }}\right| A_{2} \downarrow\right\rangle=-\left\langle A_{3} \downarrow\left|\hat{H}_{\text {so }}\right| A_{2} \uparrow\right\rangle .
$$

The remaining next-nearest-neighbor spin flipping SOCs on the $A$-sublattice, see Fig. 1 , can be connected with $\lambda_{\text {PIA }}$ by rotations $\mathcal{R}_{ \pm \frac{2 \pi}{3}}^{\hat{z}} \in D_{3 d}$. In particular, we get

$$
\begin{aligned}
&\left\langle A_{1} \uparrow\left|\hat{H}_{\mathrm{SO}}\right| A_{3} \downarrow\right\rangle \stackrel{(1 a)}{=} e^{-i \frac{2 \pi}{3}}\left\langle\mathcal{R}_{\frac{2 \pi}{3}}^{\hat{z}}\left[A_{3} \uparrow\right]\left|\hat{H}_{\mathrm{so}}\right| \mathcal{R}_{\frac{2 \pi}{3}}^{\hat{z}}\left[A_{2} \downarrow\right]\right\rangle \\
& \stackrel{(3)}{=} e^{-i \frac{2 \pi}{3} \frac{2}{3} \lambda \text { PIA }} \\
&\left\langle A_{2} \uparrow\left|\hat{H}_{\mathrm{so}}\right| A_{1} \downarrow\right\rangle \stackrel{(1 a)}{=} e^{i \frac{2 \pi}{3}}\left\langle\mathcal{R}_{-\frac{2 \pi}{3}}^{\hat{z}}\left[A_{3} \uparrow\right]\left|\hat{H}_{\mathrm{sO}}\right| \mathcal{R}_{-\frac{2 \pi}{3}}^{\hat{z}}\left[A_{2} \downarrow\right]\right\rangle \\
& \stackrel{(3)}{=} e^{i \frac{2 \pi}{3}} \frac{2}{3} \lambda_{\text {PIA }} .
\end{aligned}
$$

The SOC matrix elements on the sublattice $B$ can be obtained from the above $A$-sublattice formulas after employing the space inversion. The spin-flipping next-nearest-neighbor SOC elements for both sublattices can be compactly summarized by the following formula ${ }^{1}$

$$
\left\langle X_{m} \sigma\left|\hat{H}_{\mathrm{so}}\right| X_{n} \sigma^{\prime}\right\rangle=\left[\hat{\sigma}_{z}\right]_{X X}\left[i \hat{\boldsymbol{s}} \times \mathbf{d}_{m, n}\right]_{\sigma \sigma^{\prime}} \frac{2}{3} \lambda_{\mathrm{PIA}},
$$

where $\mathbf{d}_{m, n}=\overrightarrow{m n} /|\overrightarrow{m n}|$ is the unit vector in the horizontal ( $x y)$ plane pointing from the lattice site $n$ to the next-nearestneighbor site $m ; \hat{\boldsymbol{s}}$ stands for the array of Pauli matrices and spin projections $\sigma \neq \sigma^{\prime}$.

To summarize, the effective translationally invariant SOC Hamiltonian based on $\pi$ orbitals that respects $D_{3 d}$ symmetry and time reversal is given by

$$
\begin{aligned}
\mathcal{H}_{D_{3 d}}= & \frac{i \lambda_{\mathrm{I}}}{3 \sqrt{3}} \sum_{\sigma} \sum_{\langle m, n\rangle\rangle} v_{m, n}\left[\hat{s}_{z}\right]_{\sigma \sigma}\left|X_{m} \sigma\right\rangle\left\langle X_{n} \sigma\right| \\
& +\frac{2 \lambda_{\text {PIA }}}{3} \sum_{\sigma \neq \sigma^{\prime}} \sum_{\langle m, n\rangle\rangle}\left[\hat{\sigma}_{z}\right]_{X X}\left[i \hat{\boldsymbol{s}} \times \mathbf{d}_{m, n}\right]_{\sigma \sigma^{\prime}}\left|X_{m} \sigma\right\rangle\left\langle X_{n} \sigma^{\prime}\right| .
\end{aligned}
$$

Transforming the above SOC Hamiltonian into the Bloch form, $\mathcal{H}_{D_{3 d}}=\sum_{\mathbf{q}} \mathcal{H}_{D_{3 d}}(\mathbf{q})$, we arrive at

$$
\begin{aligned}
\mathcal{H}_{D_{3 d}}(\mathbf{q})= & \sum_{X, \sigma, \sigma^{\prime}}\left[\hat{\sigma}_{z}\right]_{X X}\left\{\lambda_{\mathrm{I}} f_{\mathrm{I}}(\mathbf{q})\left[\hat{s}_{z}\right]_{\sigma \sigma^{\prime}}+\lambda_{\mathrm{PIA}} f_{\mathrm{P}}(\mathbf{q})\left[\hat{s}_{+}\right]_{\sigma \sigma^{\prime}}\right. \\
& \left.+\lambda_{\mathrm{PIA}} \overline{f_{\mathrm{P}}(\mathbf{q})}\left[\hat{s}_{-}\right]_{\sigma \sigma^{\prime}}\right\}\left|X_{\mathbf{q}} \sigma\right\rangle\left\langle X_{\mathbf{q}} \sigma^{\prime}\right|
\end{aligned}
$$

where the structural SOC function $f_{\mathrm{I}}(\mathbf{q})$ is given by Eq. (17) and $f_{\mathrm{P}}(\mathbf{q})$ is defined as follows

$$
f_{\mathrm{P}}(\mathbf{q})=\frac{4 i}{3}\left(\sin \mathbf{q} \cdot \mathbf{R}_{1}+\mathrm{e}^{-i \frac{2 \pi}{3}} \sin \mathbf{q} \cdot \mathbf{R}_{2}+\mathrm{e}^{+i \frac{2 \pi}{3}} \sin \mathbf{q} \cdot \mathbf{R}_{3}\right) .
$$

\footnotetext{
${ }^{1}$ Space inversion interchanges sublattices, $\left[\hat{\sigma}_{z}\right]_{\mathcal{I}(X) \mathcal{I}(X)}=-\left[\hat{\sigma}_{z}\right]_{X X}$, and as well the orientation of connecting $\mathbf{d}$-vector, $\mathbf{d}_{\mathcal{I}(m), \mathcal{I}(n)}=-\mathbf{d}_{m, n}$, so in total both sign changes compensate in agreement with Eq. (31).
}

A direct inspection shows that the quasimomentum dependent spin operator (in units of $\hbar / 2$ )

$$
\operatorname{Spin}(\mathbf{q})=\hat{\sigma}_{0}\left[f_{\mathrm{P}}(\mathbf{q}) \hat{s}_{+}+\overline{f_{\mathrm{P}}(\mathbf{q})} \hat{s}_{-}+\frac{\lambda_{\mathrm{I}}}{\lambda_{\mathrm{PIA}}} f_{\mathrm{I}}(\mathbf{q}) \hat{s}_{z}\right]
$$

commutes with $\mathcal{H}_{D_{3 d}}(\mathbf{q})$. Since the orbital Hamiltonian is diagonal in spin space, the eigenstates of $H_{\text {orb }}(\mathbf{q})+\mathcal{H}_{D_{3 d}}(\mathbf{q})$ can be chosen as "spin-up" and "spin-down" states with respect to the momentum dependent quantization axis specified by the unit vector:

$$
\mathbf{n}(\mathbf{q})=\frac{\left(\operatorname{Re}\left[f_{\mathrm{P}}(\mathbf{q})\right],-\operatorname{Im}\left[f_{\mathrm{P}}(\mathbf{q})\right], \frac{\lambda_{\mathrm{I}}}{\lambda_{\mathrm{PIA}}} f_{\mathrm{I}}(\mathbf{q})\right)}{\sqrt{\left|f_{\mathrm{P}}(\mathbf{q})\right|^{2}+\frac{\lambda_{\mathrm{I}}^{2}}{\lambda_{\mathrm{PIA}}^{2}}\left|f_{\mathrm{I}}(\mathbf{q})\right|^{2}}} .
$$

Consequently, $\operatorname{Spin}(\mathbf{q}) \simeq \hat{\sigma}_{0}[\mathbf{n}(\mathbf{q}) \cdot \hat{\boldsymbol{s}}]$. It is clear that at the time-invariant momenta, i.e., at $\Gamma$ and $M$ points, $\mathbf{n}(\mathbf{q})$ is not well-defined. Hence there is not a well-defined global map from the full first Brillouin zone ( $2 d$ torus) into the $2 d$ sphere, $\mathbf{q} \mapsto \mathbf{n}(\mathbf{q})$, and thus not a well-defined global winding number. Expanding $f_{\mathrm{P}}(\mathbf{q})$ around the Dirac points, $\mathbf{q}=\tau \mathbf{K}+\mathbf{k}$, keeping the first appearing nonzero terms, we get

$$
f_{\mathrm{P}}(\tau \mathbf{K}+\mathbf{k}) \simeq-\left(i k_{x}+k_{y}\right) a_{\mathrm{L}},
$$

where $a_{\mathrm{L}}$ stands for the lattice constant. Then the effective $D_{3 d}$-invariant low-energy Hamiltonian around $\tau \mathbf{K}$ valley that includes both orbital and SOC terms is given by

$$
\begin{aligned}
\mathcal{H}_{\text {eff }}(\tau \mathbf{K}+\mathbf{k})= & \hbar v_{F}\left(\tau k_{x} \hat{\sigma}_{x}-k_{y} \hat{\sigma}_{y}\right) \hat{s}_{0}+\tau \lambda_{\mathrm{I}} \hat{\sigma}_{z} \hat{s}_{z} \\
& +\lambda_{\mathrm{PIA}} \hat{\sigma}_{z}\left(k_{x} \hat{s}_{y}-k_{y} \hat{s}_{x}\right) a_{\mathrm{L}} .
\end{aligned}
$$

Correspondingly, the momentum dependent spin quantization axis is aligned along the unit vector,

$$
\mathbf{n}(\tau \mathbf{K}+\mathbf{k})=\frac{\left(-k_{y} a_{\mathrm{L}}, k_{x} a_{\mathrm{L}}, \tau \frac{\lambda_{\mathrm{I}}}{\lambda_{\mathrm{PIA}}}\right)}{\sqrt{\left(k_{x}^{2}+k_{y}^{2}\right) a_{\mathrm{L}}^{2}+\frac{\lambda_{\mathrm{I}}^{2}}{\lambda_{\mathrm{PIA}}^{2}}}} .
$$

The eigenspectrum of $\mathcal{H}_{\text {eff }}(\tau \mathbf{K}+\mathbf{k})$-labeled by quasimomentum $\mathbf{k}$, band index $n= \pm$ and spin $\sigma$ with respect to $\mathbf{n}(\tau \mathbf{K}+\mathbf{k})$-reads

$$
\varepsilon_{n, \sigma}(\tau \mathbf{K}+\mathbf{k})=n \sqrt{\lambda_{\mathrm{I}}^{2}+\left(\hbar^{2} v_{F}^{2}+\lambda_{\mathrm{PIA}}^{2} a_{\mathrm{L}}^{2}\right)\left(k_{x}^{2}+k_{y}^{2}\right)},
$$

so the states are indeed spin degenerate as we already noticed in Eq. (30). The effect of $\lambda_{\text {PIA }}$ SOC is twofold. First, looking at the eigenspectrum, $\lambda_{\text {PIA }}$ effectively renormalizes the Fermi velocity $v_{F} \rightarrow \sqrt{v_{F}^{2}+\lambda_{\mathrm{PIA}}^{2} a_{\mathrm{L}}^{2} / \hbar^{2}}$, or equivalently in terms of the orbital nearest-neighbor hopping $t \rightarrow \sqrt{t^{2}+4 \lambda_{\text {PIA }}^{2} / 3}$. In situations when the strength of orbital hopping $t$ substantially exceeds the strength of $\lambda_{\text {PIA }}$ this effect is expected to be marginal, e.g., in silicene [77] $t \simeq 1.1 \mathrm{eV}$ and $\lambda_{\mathrm{PIA}} \simeq 0.7 \mathrm{meV}$, however, in germanene [77], $t \simeq 0.9 \mathrm{eV}$ and $\lambda_{\text {PIA }} \simeq 10.7 \mathrm{meV}$ and hence the renormalization of the orbital hopping should be more pronounced. Second, $\lambda_{\text {PIA }}$ introduces a nontrivial spin-orbit field in the $k$ space, $\mathbf{n}(\mathbf{q}) \cdot \hat{\boldsymbol{s}}$, that gives rise to the in plane component of the spin-expectation value, see Fig. 4. 


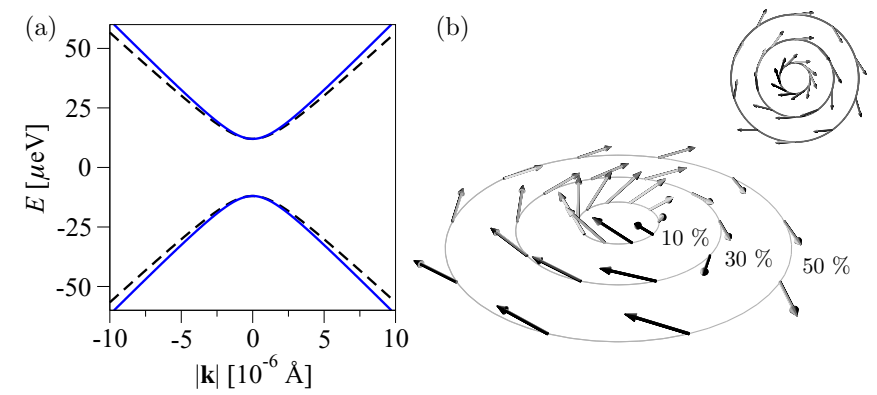

FIG. 4. Electronic band structure and spin-orbit field around the $K$-point in the presence of $D_{3 d}$ invariant SOC Hamiltonian. (a) Electronic band structure for $t=2.6 \mathrm{eV}, \lambda_{\mathrm{I}}=12 \mu \mathrm{eV}$, and $\lambda_{\text {PIA }}=$ $0.1 \mathrm{eV}$ (black dashed) and $\lambda_{\text {PIA }}=1 \mathrm{eV}$ (blue solid), respectively, showing the effect of renormalization of Fermi velocity. (b) $D_{3 d}$ spin-orbit field around the Dirac point along the circles with radius $10 \%, 30 \%$, and $50 \%$ of $\overline{\mathrm{KM}}$-distance for $t=2.6 \mathrm{eV}, \lambda_{\mathrm{I}}=12 \mu \mathrm{eV}$, and $\lambda_{\text {PIA }}=60 \mu \mathrm{eV}$. The inset shows a top view of the spin-orbit field. The circular low-energy symmetry changes to the triangular one and the $z$ component of the spin-orbit field becomes suppressed when moving away from the Dirac point.

\section{2. $D_{3 h}$ case: $\lambda_{\mathrm{I}}^{\mathrm{A}}$ and $\lambda_{\mathrm{I}}^{\mathrm{B}}$ couplings}

Hexagonal boron nitride is a prototype of a planar hexagonal structure that consists of two nonequivalent interpenetrating triangular lattices - in our particular example, composed of borons and nitrogens, respectively. Since the horizontal reflection $\Sigma_{h}^{x y}$ belongs to $D_{3 h}$, spin-flipping SOC mediated hoppings are not allowed according to the no-go argument (see Sec. III B 1). Similarly, the vertical mirror reflection $\Sigma_{v}^{y z}$ belongs to $D_{3 h}$ and hence by the assertion (see Sec. III B 3) there are neither spin-conserving nearest-neighbor SOCs. Therefore we are left with the intrinsic-next-nearest-neighbor spinconserving SOC - terms only. The broken sublattice symmetry can not further constrain the intrinsic SOCs and hence they become sublattice dependent, i.e., $\lambda_{\mathrm{I}}^{\mathrm{A}} \neq \lambda_{\mathrm{I}}^{\mathrm{B}}$. Motivated by the previous analysis and knowing that they are purely imaginary we define them via the formulas

$$
\begin{aligned}
& \frac{i \lambda_{\mathrm{I}}^{\mathrm{A}}}{3 \sqrt{3}}=\left\langle A_{3} \uparrow\left|\hat{H}_{\mathrm{so}}\right| A_{2} \uparrow\right\rangle, \\
& \frac{i \lambda_{\mathrm{I}}^{\mathrm{B}}}{3 \sqrt{3}}=\left\langle B_{2} \downarrow\left|\hat{H}_{\mathrm{so}}\right| B_{3} \downarrow\right\rangle ;
\end{aligned}
$$

for the atomic sites configuration see Fig. 1. In analogy with Eq. (12), the $D_{3 h}$ invariant SOC Hamiltonian reads

$$
\begin{aligned}
\mathcal{H}_{D_{3 h}}= & \frac{i \lambda_{\mathrm{I}}^{\mathrm{A}}}{3 \sqrt{3}} \sum_{\sigma} \sum_{\langle m, n\rangle\rangle} v_{m, n}\left[\hat{s}_{z}\right]_{\sigma \sigma}\left|A_{m} \sigma\right\rangle\left\langle A_{n} \sigma\right| \\
& +\frac{i \lambda_{\mathrm{I}}^{\mathrm{B}}}{3 \sqrt{3}} \sum_{\sigma} \sum_{\langle m, n\rangle\rangle} v_{m, n}\left[\hat{s}_{z}\right]_{\sigma \sigma}\left|B_{m} \sigma\right\rangle\left\langle B_{n} \sigma\right| .
\end{aligned}
$$

Contrary to the $D_{6 h}$ case, the lack of space inversion symmetry $\mathcal{I}$ in $D_{3 h}$-hence two different values of $\lambda_{\mathrm{I}}^{\mathrm{A}}$ and $\lambda_{\mathrm{I}}^{\mathrm{B}}$-causes spin splitting of the band structure, see Fig. 5.

The low-energy Bloch representation of $\mathcal{H}_{\mathrm{orb}}(\tau \mathbf{K}+\mathbf{k})+$ $\mathcal{H}_{D_{3 h}}(\tau \mathbf{K}+\mathbf{k})$ can be easily deduced from Eq. (25) when properly substituting $\lambda_{\mathrm{I}}$ by its sublattice resolved counterparts
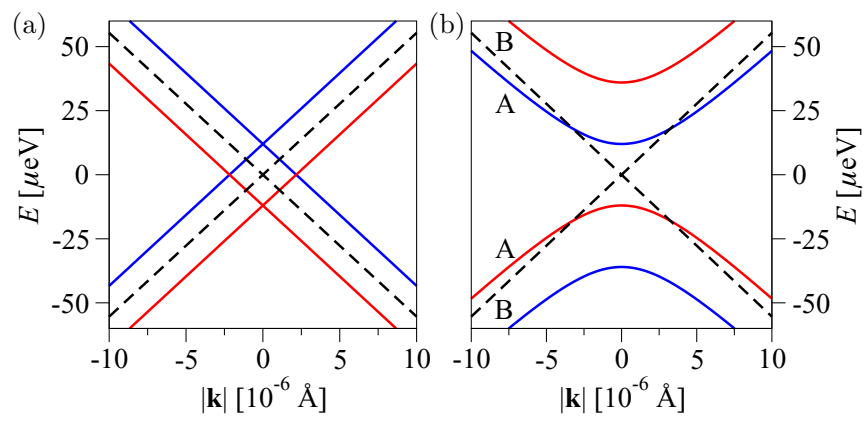

FIG. 5. Electronic band structure around the $K$-point in the presence of $D_{3 h}$ invariant SOC Hamiltonian without staggered potential $\Delta$ : for $t=2.6 \mathrm{eV}$ and $\lambda_{\mathrm{I}}^{\mathrm{A}}=12 \mu \mathrm{eV}$ and (a) $\lambda_{\mathrm{I}}^{\mathrm{B}}=-\lambda_{\mathrm{I}}^{\mathrm{A}}$ and (b) $\lambda_{\mathrm{I}}^{\mathrm{B}}=3 \lambda_{\mathrm{I}}^{\mathrm{A}}$. Blue and red lines indicate bands with up and down spin projections, respectively. Label A or B at the given band indicates which sublattice is dominantly occupied by electronic states at that band, assuming their momenta are close to the Dirac point. For comparison, the black dashed lines display the energy dispersion of the pristine graphene without SOC and the staggered $\Delta$.

$\lambda_{\mathrm{I}}^{\mathrm{A}}$ and $\lambda_{\mathrm{I}}^{\mathrm{B}}$. The result is as follows:

$$
\begin{aligned}
\mathcal{H}_{\mathrm{eff}}(\tau \mathbf{K}+\mathbf{k})= & \hbar v_{F}\left(\tau k_{x} \hat{\sigma}_{x}-k_{y} \hat{\sigma}_{y}\right) \hat{s}_{0}+\Delta \hat{\sigma}_{z} \hat{s}_{0} \\
& +\frac{\tau}{2}\left[\lambda_{\mathrm{I}}^{\mathrm{A}}\left(\hat{\sigma}_{z}+\hat{\sigma}_{0}\right)+\lambda_{\mathrm{I}}^{\mathrm{B}}\left(\hat{\sigma}_{z}-\hat{\sigma}_{0}\right)\right] \hat{s}_{z} .
\end{aligned}
$$

Contrary to the previous cases, the broken sublattice symmetry allows also a new term in the orbital Hamiltonian $\mathcal{H}_{\text {orb }}$ - the second term in the first line parameterized by the so called staggered potential $\Delta$. The two inequivalent sublattices can possess different on-site energies and their difference equals $2 \Delta$. Similarly as in the $D_{6 h}$ case, the spin operator $\hat{\sigma}_{0} \hat{s}_{z}$ commutes with $\mathcal{H}_{\text {eff }}$ allowing us to label its eigenstates with the spin up and spin down entries. The eigenspectrum of $\mathcal{H}_{\text {eff }}(\tau \mathbf{K}+\mathbf{k})-$ labeled by the quasimomentum $\mathbf{k}$, conduction/valence band index $n=+/-$ and $\operatorname{spin} \sigma=\{\uparrow, \downarrow\}=\{+1,-1\}$ with respect to $\hat{s}_{z}$ reads

$$
\begin{aligned}
\varepsilon_{n, \sigma}(\tau \mathbf{K}+\mathbf{k})= & \frac{\sigma}{2}\left(\lambda_{\mathrm{I}}^{\mathrm{A}}-\lambda_{\mathrm{I}}^{\mathrm{B}}\right) \\
& +n \sqrt{\left[\Delta+\frac{\sigma}{2}\left(\lambda_{\mathrm{I}}^{\mathrm{A}}+\lambda_{\mathrm{I}}^{\mathrm{B}}\right)\right]^{2}+\hbar^{2} v_{F}^{2}\left(k_{x}^{2}+k_{y}^{2}\right)} .
\end{aligned}
$$

The band structure visualization of the SOC induced splittings in the presence of staggered $\Delta$ are displayed in Fig. 6 . Direct analysis of Eq. (53) shows that there are two distinct spectral cases - an insulating (gapped) and a band-inverted (gapless) one. The criteria to get spectral band-inversion are sign $\lambda_{\mathrm{I}}^{\mathrm{A}} \neq$ $\operatorname{sign} \lambda_{\mathrm{I}}^{\mathrm{B}}$ and $|\Delta|<\max \left(\left|\lambda_{\mathrm{I}}^{\mathrm{A}}\right|,\left|\lambda_{\mathrm{I}}^{\mathrm{B}}\right|\right)$.

\section{3. $C_{6 v}$ case $: \lambda_{\mathrm{I}}, \lambda_{\mathrm{R}}$, and $\lambda_{\mathrm{PIA}}$ couplings}

Graphene in an external transverse electric field or graphene disposed on a substrate is a prototype of the structure with $C_{6 v}$ structural symmetry. In this case the sublattices remain equivalent - the rotation $\mathcal{R}_{\pi / 3}^{\hat{z}}$ that interchanges them belongs to the point group. However, we lose all the structural symmetries flipping the orientation of the transverse $z$-axis. According to the arguments in Sec. III B, lack of space 

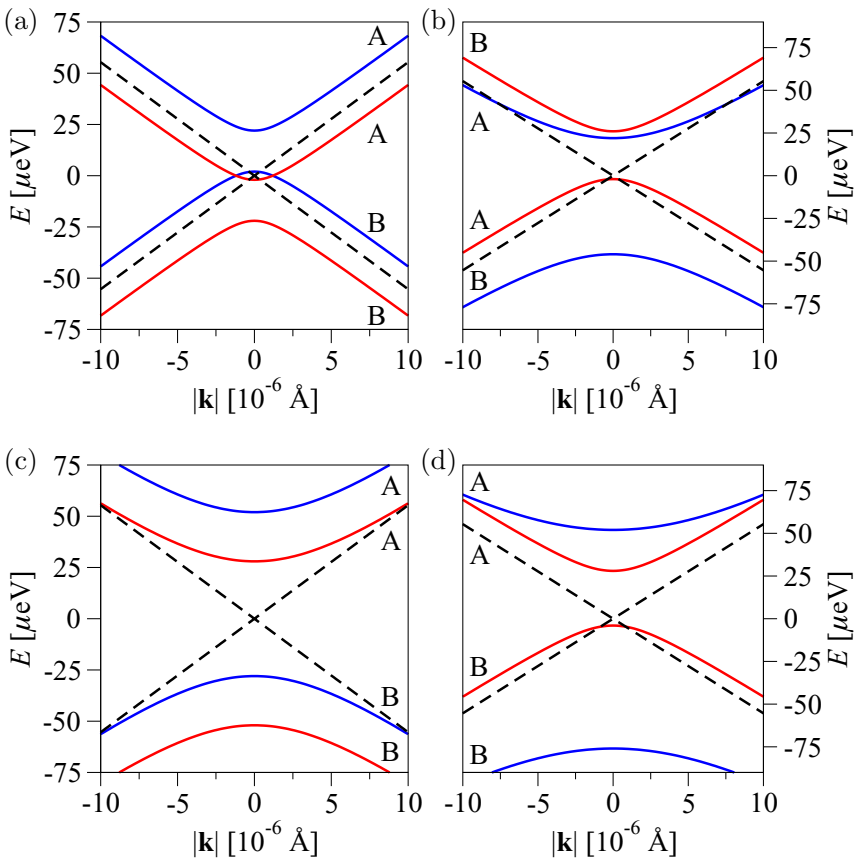

FIG. 6. Electronic band structure around the $K$-point in the presence of $D_{3 h}$ invariant SOC Hamiltonian and staggered potential $\Delta$ for $t=2.6 \mathrm{eV}$ and $\lambda_{\mathrm{I}}^{\mathrm{A}}=12 \mu \mathrm{eV}$ and (a) $\lambda_{\mathrm{I}}^{\mathrm{B}}=-\lambda_{\mathrm{I}}^{\mathrm{A}}$ and $\Delta=10 \mu \mathrm{eV}$, (b) $\lambda_{\mathrm{I}}^{\mathrm{B}}=3 \lambda_{\mathrm{I}}^{\mathrm{A}}$ and $\Delta=10 \mu \mathrm{eV}$, (c) $\lambda_{\mathrm{I}}^{\mathrm{B}}=-\lambda_{\mathrm{I}}^{\mathrm{A}}$ and $\Delta=40 \mu \mathrm{eV}$, and (d) $\lambda_{\mathrm{I}}^{\mathrm{B}}=3 \lambda_{\mathrm{I}}^{\mathrm{A}}$ and $\Delta=40 \mu \mathrm{eV}$, respectively. For $\operatorname{sign} \lambda_{\mathrm{I}}^{\mathrm{A}} \neq \operatorname{sign} \lambda_{\mathrm{I}}^{\mathrm{B}}$, the increased value of the staggered potential drives the band-inverted structure (a) to the insulating one (c). Blue and red lines indicate bands with up and down spin projections, respectively, and the dashed lines display the orbital band structure of pristine graphene. Label A or B at the given band indicates which sublattice is dominantly occupied by electronic states at that band, assuming their momenta are close to the Dirac point.

inversion $\mathcal{I}$ and horizontal reflection $\Sigma_{h}^{x y}$ can not prevent the system from spin-flip SOC hoppings among nearest- and next-nearest neighbors. Contrary to that, $\Sigma_{v}^{y z}$ inhibits the spin-conserving nearest-neighbor SOCs, but allows intrinsicnext-nearest-neighbor-SOCs. From this finger counting symmetry analysis and the no-go arguments we know that the $C_{6 v}$ invariant SOC Hamiltonian would potentially host three couplings: $\lambda_{\mathrm{I}}$ and $\lambda_{\mathrm{PIA}}$ - the terms analogous with the already discussed $D_{3 d}$ case, see Eq. (33) and (34) - and the new spin-flipping term $\lambda_{R}$ acting between the nearest neighbors. Conventionally the latter is called Rashba SOC [88] and in terms of a SOC matrix element it can be defined as follows,

$$
\frac{2}{3} i \lambda_{\mathrm{R}} \equiv\left\langle A_{2} \uparrow\left|\hat{H}_{\mathrm{so}}\right| B_{3} \downarrow\right\rangle \stackrel{(5)}{=}-\left\langle B_{3} \uparrow\left|\hat{H}_{\mathrm{so}}\right| A_{2} \downarrow\right\rangle
$$

In the above definition we have already employed the purely imaginary character of the coupling. Applying the dihedral reflection $\Sigma_{d}^{x z}$ to the defining matrix element we obtain,

$$
\begin{aligned}
\left\langle A_{2} \uparrow\left|\hat{H}_{\mathrm{so}}\right| B_{3} \downarrow\right\rangle & =\left\langle\Sigma_{d}^{x z}\left[B_{3} \downarrow\right]\left|\hat{H}_{\mathrm{so}}\right|-\Sigma_{d}^{x z}\left[A_{2} \uparrow\right]\right\rangle \\
& \stackrel{(3)}{=}-\left\langle B_{3} \downarrow\left|\hat{H}_{\mathrm{so}}\right| A_{2} \uparrow\right\rangle=-\overline{\left\langle A_{2} \uparrow\left|\hat{H}_{\mathrm{so}}\right| B_{3} \downarrow\right\rangle},
\end{aligned}
$$

what is indeed what we wanted to show. In analogy with Eq. (39) we can also write a compact formula for any nearest-neighbor spin-flipping matrix element,

$$
\left\langle X_{m} \sigma\left|\hat{H}_{\mathrm{so}}\right| X_{n}^{\prime} \sigma^{\prime}\right\rangle=\left[\hat{\boldsymbol{s}} \times \mathbf{d}_{m, n}\right]_{\sigma \sigma^{\prime}} \frac{2}{3} i \lambda_{\mathrm{R}},
$$

where, $\mathbf{d}_{m, n}=\overrightarrow{m n} /|\overrightarrow{m n}|$ is the unit vector in the horizontal ( $x y)$ plane pointing from lattice site $n$ to nearest-neighbor site $m$ and $\sigma \neq \sigma^{\prime}$, and $X$ and $X^{\prime}$ refer to the opposite sublattices.

Let us emphasize that contrary to $D_{3 d}$ case the point group $C_{6 v}$ lacks space inversion. Therefore the PIA analogs of Eq. (31) in $C_{6 v}$ case reads

$$
\begin{gathered}
\left\langle A_{i} \sigma\left|\hat{H}_{\mathrm{so}}\right| A_{j} \sigma^{\prime}\right\rangle \stackrel{(1 a)}{=}\left\langle e^{i \frac{\pi}{2} \sigma} \mathcal{R}_{\pi}^{\hat{z}}\left[B_{i} \sigma\right]\left|\hat{H}_{\mathrm{so}}\right| e^{i \frac{\pi}{2} \sigma^{\prime}} \mathcal{R}_{\pi}^{\hat{z}}\left[B_{j} \sigma^{\prime}\right]\right\rangle \\
\stackrel{(3)}{=} e^{i \frac{\pi}{2}\left(\sigma^{\prime}-\sigma\right)}\left\langle B_{i} \sigma\left|\hat{H}_{\mathrm{so}}\right| B_{j} \sigma^{\prime}\right\rangle=-\left\langle B_{i} \sigma\left|\hat{H}_{\mathrm{so}}\right| B_{j} \sigma^{\prime}\right\rangle,
\end{gathered}
$$

and the analog of (39) is as follows:

$$
\left\langle X_{m} \sigma\left|\hat{H}_{\mathrm{so}}\right| X_{n} \sigma^{\prime}\right\rangle=\left[i \hat{\boldsymbol{s}} \times \mathbf{d}_{m, n}\right]_{\sigma \sigma^{\prime}} \frac{2}{3} \lambda_{\text {PIA }} .
$$

So the general $C_{6 v}$ invariant SOC Hamiltonian based on $\pi$-states, time reversal and translational invariance reads,

$$
\begin{aligned}
\mathcal{H}_{C_{6 v}}= & \frac{i \lambda_{\mathrm{I}}}{3 \sqrt{3}} \sum_{\sigma} \sum_{\langle m, n\rangle\rangle} v_{m, n}\left[\hat{s}_{z}\right]_{\sigma \sigma}\left|X_{m} \sigma\right\rangle\left\langle X_{n} \sigma\right| \\
& +\frac{2 \lambda_{\mathrm{PIA}}}{3} \sum_{\sigma \neq \sigma^{\prime}} \sum_{\langle m, n\rangle\rangle}\left[i \hat{\boldsymbol{s}} \times \mathbf{d}_{m, n}\right]_{\sigma \sigma^{\prime}}\left|X_{m} \sigma\right\rangle\left\langle X_{n} \sigma^{\prime}\right| \\
& +\frac{2 i \lambda_{\mathrm{R}}}{3} \sum_{\sigma \neq \sigma^{\prime}} \sum_{\langle m, n\rangle}\left[\hat{\boldsymbol{s}} \times \mathbf{d}_{m, n}\right]_{\sigma \sigma^{\prime}}\left|X_{m} \sigma\right\rangle\left\langle X_{n} \sigma^{\prime}\right| .
\end{aligned}
$$

The first and the last term in $\mathcal{H}_{C_{6 v}}$ are the well known SOC terms from the seminal papers of Kane and Mele [32,88]. However, the staggered potential $\Delta$ added and considered by them in the orbital part [see Eq. (1) in Ref. [88]] is in fact not compatible with the $C_{6 v}$ symmetry, but rather the $C_{3 v}$ one discussed in the next section. What is more striking is the presence of the second- $\lambda_{\text {PIA }}$ SOC term-which seems to be generally overseen by the community. Readers can easily convince themselves that there are not enough symmetries in $C_{6 v}$ that can cancel its appearance in $\mathcal{H}_{C_{6 v}}$. Indeed, to map the real matrix element $\left\langle A_{3} \uparrow\left|\hat{H}_{\text {so }}\right| A_{2} \downarrow\right\rangle \sim \lambda_{\text {PIA }}$, Eq. (34), to " \pm itself" within the pool of $C_{6 v}$ symmetries, one can use respectively the vertical, $\Sigma_{v}^{y z}$, and dihedral, $\Sigma_{d}^{x z}$, reflectionsboth flip spins - and accompany them by the rotation $\mathcal{R}_{\pi}^{\hat{z}}$, see Figs. 1 and 3. Since $\mathcal{R}_{\pi}^{\hat{z}}=\Sigma_{v}^{y z} \circ \Sigma_{d}^{x z}$, the composition $\mathcal{S}=\mathcal{R}_{\pi}^{\hat{z}} \circ \Sigma_{d}^{x z} \circ \Sigma_{v}^{y z}$ is the identity in the orbital and also in the spin space and hence $\mathcal{S}:\left|A_{i} \sigma\right\rangle \rightarrow\left|A_{i} \sigma\right\rangle$. So at the end $\left\langle A_{3} \uparrow\left|\hat{H}_{\mathrm{so}}\right| A_{2} \downarrow\right\rangle=+\left\langle A_{3} \uparrow\left|\hat{H}_{\mathrm{so}}\right| A_{2} \downarrow\right\rangle$ what gives no constraint on $\lambda_{\text {PIA. }}$.

The first (intrinsic) SOC term in $\mathcal{H}_{C_{6 v}}$, Eq. (59)—as we have discussed earlier-is not causing SOC splitting of the electronic band structure. The band SOC splitting is solely due to the space inversion breaking term-Rashba SOC $\lambda_{R}$, that is accompanied by the principal-plane mirror asymmetry termPIA SOC $\lambda_{\text {PIA }}$. One can anticipate this fact either directly from eigenspectrum (discussed below) or from the generally valid argument of Bychkov and Rashba [87]. They showed that a SOC induced band spin-splitting would appear in systems with a single high-symmetry (at least three-fold) axis, in our case 

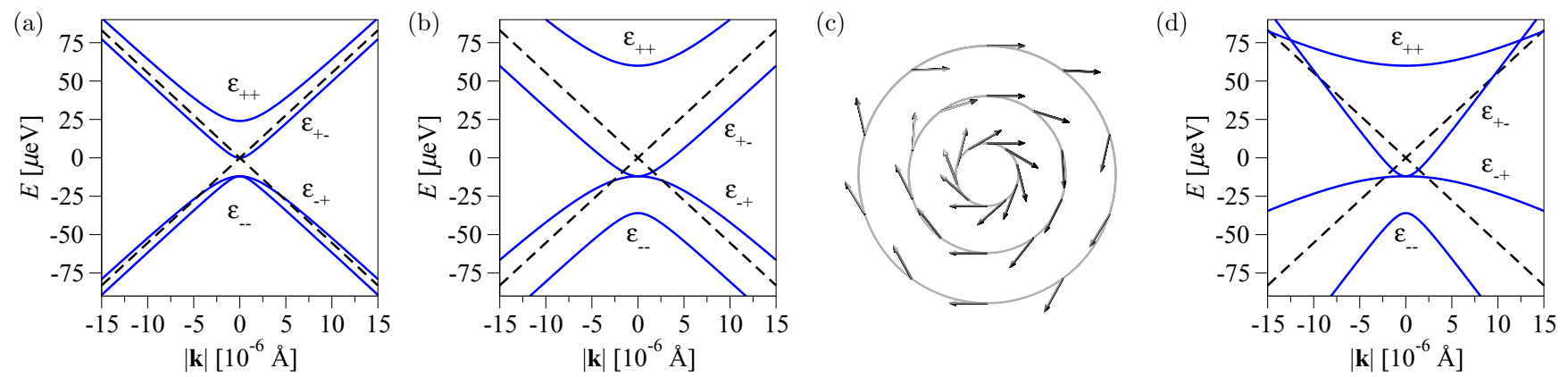

FIG. 7. Electronic band structure and spin-orbit field around the $K$-point in the presence of $C_{6 v}$ invariant SOC Hamiltonian for fixed $t=2.6 \mathrm{eV}$ and $\lambda_{\mathrm{I}}=12 \mu \mathrm{eV}$, and different values of $\lambda_{\mathrm{R}}$ and $\lambda_{\text {PIA }}$ SOC parameters. In panel (a) $\lambda_{\mathrm{R}}=6 \mu \mathrm{eV}<\lambda_{\mathrm{I}}$ (gapped) and $\lambda_{\text {PIA }}=0$; in panel (b) $\lambda_{\mathrm{R}}=24 \mu \mathrm{eV}>\lambda_{\mathrm{I}}$ (gapless) and $\lambda_{\text {PIA }}=0$. Panel (c) displays the spin-orbit field $\mathbf{n}(\mathbf{q})$ around $K$-point for the energy band $\varepsilon_{--}$in figure (b), circles radii correspond to $10 \%, 30 \%$ and $50 \%$ of $\overline{\mathrm{KM}}$-distance. In panel (d) $\lambda_{\mathrm{R}}=24 \mu \mathrm{eV}>\lambda_{\mathrm{I}}$ (gapless) and $\lambda_{\text {PIA }}=1 \mathrm{eV}$, a non-zero value of $\lambda_{\text {PIA }}$ renormalizes the Fermi velocities $v_{F}$ of different spin-split sub-bands $\varepsilon_{n, n^{\prime}}(\mathbf{K}+\mathbf{k})$. For $\Omega>0$, see Eq. (66), $\lambda_{\text {PIA }}$ causes a band crossing in the conduction (displayed case) or valence band. For comparison, the black dashed lines display the energy dispersion of the pristine graphene without SOC.

the transverse $z$-axis, and an invariant vector along this axis, in our case the transverse electric field or the outward direction from the surface, what is exactly the case of $C_{6 v}$ group and its subgroups.

In what follows we transform the $C_{6 v}$-invariant SOC Hamiltonian into the Bloch form, $\mathcal{H}_{C_{6 v}}=\sum_{\mathbf{q}} \mathcal{H}_{C_{6 v}}(\mathbf{q})$. The intrinsic term in $\mathcal{H}_{C_{6 v}}(\mathbf{q})$ is identical with $\mathcal{H}_{D_{6 h}}(\mathbf{q})$, see Eq. (16), the PIA term entering $\mathcal{H}_{C_{6 v}}(\mathbf{q})$ can be deduced from the corresponding term in $\mathcal{H}_{D_{3 d}}(\mathbf{q})$, Eq. (41), omitting there $\left[\hat{\sigma}_{z}\right]_{X X}$. For that reason we write here explicitly only the Rashba part

$$
\begin{aligned}
\mathcal{H}_{\mathrm{R}}(\mathbf{q})= & i \lambda_{\mathrm{R}} \sum_{\sigma, \sigma^{\prime}} \sum_{X, X^{\prime}}\left\{[ \hat { \sigma } _ { + } ] _ { X X ^ { \prime } } \left(f_{\mathrm{R}}(\mathbf{q})\left[\hat{s}_{+}\right]_{\sigma \sigma^{\prime}}\right.\right. \\
& \left.+\overline{f_{\mathrm{R}}(-\mathbf{q})}\left[\hat{s}_{-}\right]_{\sigma \sigma^{\prime}}\right)-\left[\hat{\sigma}_{-}\right]_{X X^{\prime}}\left(\overline{f_{\mathrm{R}}(\mathbf{q})}\left[\hat{s}_{-}\right]_{\sigma \sigma^{\prime}}\right. \\
& \left.\left.+f_{\mathrm{R}}(-\mathbf{q})\left[\hat{s}_{+}\right]_{\sigma \sigma^{\prime}}\right)\right\}\left|X_{\mathbf{q}} \sigma\right\rangle\left\langle X_{\mathbf{q}}^{\prime} \sigma^{\prime}\right| .
\end{aligned}
$$

The Rashba SOC structural function is given as follows,

$$
f_{\mathrm{R}}(\mathbf{q})=\frac{2}{3}\left\{1+\mathrm{e}^{-i \frac{2 \pi}{3}} \mathrm{e}^{-i \mathbf{q} \cdot \mathbf{R}_{3}}+\mathrm{e}^{i \frac{2 \pi}{3}} \mathrm{e}^{i \mathbf{q} \cdot \mathbf{R}_{2}}\right\},
$$

and the sublattice raising/lowering operators are defined by $\hat{\sigma}_{ \pm}=\frac{1}{2}\left(\hat{\sigma}_{x} \pm i \hat{\sigma}_{y}\right)$. In our sublattice convention we particulary have $\left[\hat{\sigma}_{+}\right]_{A B}=1=\left[\hat{\sigma}_{-}\right]_{B A}$ and $\left[\hat{\sigma}_{+}\right]_{B A}=0=\left[\hat{\sigma}_{-}\right]_{A B}$. It is worth to mention that there does not exist a simple SOC field $\mathbf{n}(\mathbf{q}) \cdot \hat{\boldsymbol{s}}$ such that the operator $\hat{\sigma}_{0}[\mathbf{n}(\mathbf{q}) \cdot \hat{\boldsymbol{s}}]$ commutes with $\mathcal{H}_{C_{6 v}}(\mathbf{q})$.

The low energy expansion $f_{\mathrm{R}}(\tau \mathbf{K}+\mathbf{k})$ to the first order in k can be summarized by,

$$
f_{\mathrm{R}}(\tau \mathbf{K}+\mathbf{k})= \begin{cases}2+i \frac{2}{\sqrt{3}} k_{y} a_{\mathrm{L}} & \text { for }+\mathbf{K}, \\ -\frac{1}{\sqrt{3}} k_{x} a_{\mathrm{L}}-i \frac{1}{\sqrt{3}} k_{y} a_{\mathrm{L}} & \text { for }-\mathbf{K} .\end{cases}
$$

Since Rashba SOC is off-diagonal in spin and sublattice spaces, it is common to approximate $f_{\mathrm{R}}(\tau \mathbf{K}+\mathbf{k})$ by $f_{\mathrm{R}}(\tau \mathbf{K})$. Doing so we get the effective $C_{6 v}$-invariant low energy Hamiltonian,

$$
\begin{aligned}
\mathcal{H}_{\text {eff }}(\tau \mathbf{K}+\mathbf{k})= & \hbar v_{F}\left(\tau k_{x} \hat{\sigma}_{x}-k_{y} \hat{\sigma}_{y}\right) \hat{s}_{0}+\tau \lambda_{\mathrm{I}} \hat{\sigma}_{z} \hat{s}_{z} \\
& +\lambda_{\mathrm{PIA}} \hat{\sigma}_{0}\left(k_{x} \hat{s}_{y}-k_{y} \hat{s}_{x}\right) a_{\mathrm{L}}-\lambda_{\mathrm{R}}\left(\tau \hat{\sigma}_{x} \hat{s}_{y}+\hat{\sigma}_{y} \hat{s}_{x}\right) .
\end{aligned}
$$

whose eigenspectrum labeled by $n= \pm$ and $n^{\prime}= \pm$ reads,

$$
\begin{aligned}
& \varepsilon_{n, n^{\prime}}(\tau \mathbf{K}+\mathbf{k}) \\
& =n^{\prime} \lambda_{\mathrm{R}}+n \sqrt{\left(\lambda_{\mathrm{I}}+n^{\prime} \lambda_{\mathrm{R}}\right)^{2}+\left(\hbar v_{F}-n^{\prime} \lambda_{\mathrm{PIA}} a_{\mathrm{L}}\right)^{2}\left(k_{x}^{2}+k_{y}^{2}\right) .}
\end{aligned}
$$

Similarly as before, $\mathbf{k}$ is the quasi-momentum measured with respect to the given $\tau \mathbf{K}$-valley, $n= \pm$ stands for the conduction and valence bands, respectively, and the index $n^{\prime}= \pm$ stands for the spin polarization. The spin expectation value - spin-orbit field $\mathbf{n}(\mathbf{q})$ - at the given $\mathbf{q}$ and the band indices $n$ and $n^{\prime}$ can be computed from the normalized eigenstates $\left|\mathbf{q}, n, n^{\prime}\right\rangle$ via $\mathbf{n}(\mathbf{q})=\left\langle\mathbf{q}, n, n^{\prime}|\hat{\boldsymbol{s}}| \mathbf{q}, n, n^{\prime}\right\rangle$. The general formula is too complex and therefore we present only a result for the low energy eigenstates $\left|\tau \mathbf{K}+\mathbf{k}, n, n^{\prime}\right\rangle$ of Eq. (63) around the $\tau \mathbf{K}$-valley:

$$
\left(\begin{array}{c}
\left\langle\hat{s}_{x}\right\rangle \\
\left\langle\hat{s}_{y}\right\rangle \\
\left\langle\hat{s}_{z}\right\rangle
\end{array}\right)=\alpha_{n, n^{\prime}} \cdot \beta_{n^{\prime}}\left(\begin{array}{c}
-n^{\prime} k_{y} / k \\
+n^{\prime} k_{x} / k \\
0
\end{array}\right)
$$

Here $\quad \alpha_{n, n^{\prime}}=\operatorname{sign}\left(\tau \lambda_{\mathrm{I}}+2 n^{\prime} \lambda_{\mathrm{R}}-\varepsilon_{n, n^{\prime}}\right), \quad \beta_{n^{\prime}}=\operatorname{sign}\left(\hbar v_{F}-\right.$ $\left.n^{\prime} \lambda_{\mathrm{PIA}} a_{\mathrm{L}}\right), k \equiv \sqrt{k_{x}^{2}+k_{y}^{2}}$, and $\varepsilon_{n, n^{\prime}}$ stands as a shorthand for eigenenergy $\varepsilon_{n, n^{\prime}}(\tau \mathbf{K}+\mathbf{k})$, see Eq. (64). For the visualization of the band structure and the spin-orbit field texture see Fig. 7. It is worth to emphasize that at the Dirac points the two eigenvalues out of four become always degenerate. For example, for $\lambda_{\mathrm{I}}>\lambda_{\mathrm{R}}>0$ we have $\varepsilon_{-,-}(\tau \mathbf{K})=\varepsilon_{-,+}(\tau \mathbf{K})=-\lambda_{\mathrm{I}}$ for the valence bands, and $\varepsilon_{+, \mp}(\tau \mathbf{K})=\lambda_{\mathrm{I}} \mp 2 \lambda_{\mathrm{R}}$ for conduction ones; the spectrum possesses SOC induced gap with value $2\left(\lambda_{\mathrm{I}}-\right.$ $\left.\lambda_{\mathrm{R}}\right)$. For $\lambda_{\mathrm{R}}>\lambda_{\mathrm{I}}>0$ we have $\varepsilon_{-,+}(\tau \mathbf{K})=\varepsilon_{+,-}(\tau \mathbf{K})=-\lambda_{\mathrm{I}}$, i.e. one valence and one conduction bands touch each other, and hence the spectral gap closes, the two remaining bands are starting from energies $\varepsilon_{\mp,-}(\tau \mathbf{K})=\mp 2 \lambda_{\mathrm{R}}+\lambda_{\mathrm{I}}$. The case $\lambda_{\mathrm{I}}=\lambda_{\mathrm{R}}>0$ is critical, the spectrum changes from the gaped to gapless and we have a triple degeneracy $\varepsilon_{-,-}(\tau \mathbf{K})=$ $\varepsilon_{-,+}(\tau \mathbf{K})=\varepsilon_{+,-}(\tau \mathbf{K})=-\lambda_{\mathrm{I}}$. Moving away from the Dirac points the PIA SOC starts to manifest, see Eq. (64). For the given band index $n$ the two spin split branches $n^{\prime}= \pm$ with energies $\varepsilon_{n, \pm}(\tau \mathbf{K}+\mathbf{k})$ possess different Fermi velocities 

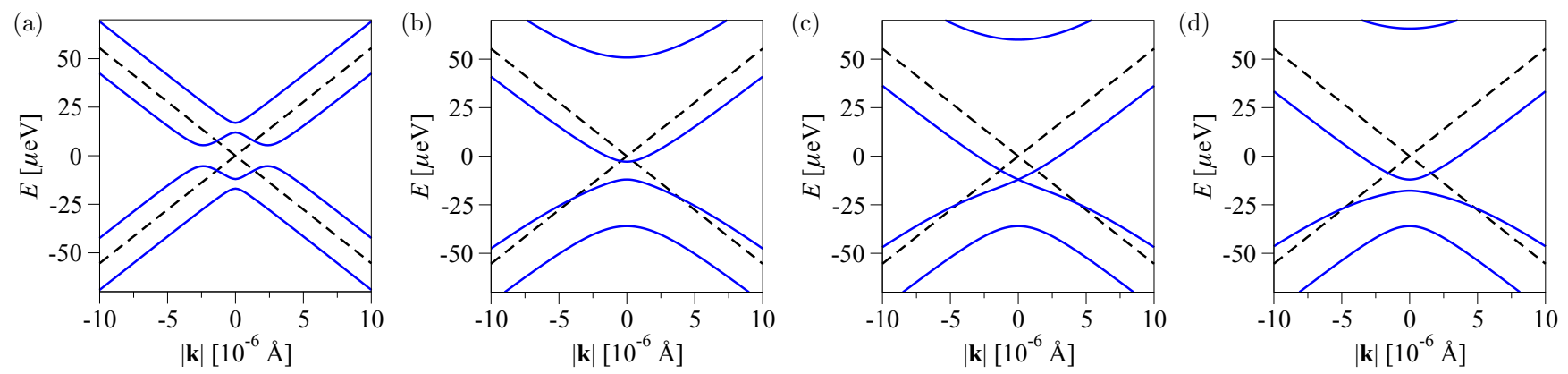

FIG. 8. Electronic band structure around the $K$-point in the presence of $C_{3 v}$ invariant SOC Hamiltonian for fixed $t=2.6 \mathrm{eV}$, staggered potential $\Delta=0$ and $\lambda_{\mathrm{I}}^{\mathrm{A}}=12 \mu \mathrm{eV}$, and different values of SOC parameters. In panel (a) $\lambda_{\mathrm{I}}^{\mathrm{B}}=-\lambda_{\mathrm{I}}^{\mathrm{A}}$ and $\lambda_{\mathrm{R}}=6 \mu \mathrm{eV}$, the band inversion from Fig. 5(a) is lifted due to presence of finite Rashba SOC $\lambda_{\mathrm{R}}$. In panels (b)-(d) $\lambda_{\mathrm{I}}^{\mathrm{B}}=3 \lambda_{\mathrm{I}}^{\mathrm{A}}$, gradually growing $\lambda_{\mathrm{R}}, 6 \mu \mathrm{eV}<\sqrt{2} \lambda_{\mathrm{I}}^{\mathrm{A}}<20 \mu \mathrm{eV}$, drives the trivial band structure displayed at Fig. 5(b) from gapped (b) to gapless (c) and gapped again (d). For comparison, the black dashed lines display the energy dispersion of the pristine graphene without SOC.

$$
\begin{aligned}
& v_{F \pm}=\sqrt{v_{F}^{2} \mp \lambda_{\text {PIA }}^{2} a_{\mathrm{L}}^{2} / \hbar^{2}} \text {. If } \Omega, \text { defined as follows } \\
& \Omega=1+2 \frac{\lambda_{\mathrm{I}}}{\lambda_{\mathrm{R}}} \frac{\lambda_{\mathrm{PIA}} a_{\mathrm{L}}}{\hbar v_{F}}+\left(\frac{\lambda_{\mathrm{PIA}} a_{\mathrm{L}}}{\hbar v_{F}}\right)^{2} \approx 1+2 \frac{\lambda_{\mathrm{I}}}{\lambda_{\mathrm{R}}} \frac{\lambda_{\text {PIA }} a_{\mathrm{L}}}{\hbar v_{F}},
\end{aligned}
$$

is greater then zero, the two spin-split bands $\varepsilon_{n, \pm}(\tau \mathbf{K}+\mathbf{k})$ cross along a circle in $\mathrm{k}$-space with a center at $\tau \mathbf{K}$ and radius

$$
k_{\mathrm{cr}}=\left|\frac{\lambda_{\mathrm{R}}}{\lambda_{\mathrm{PIA}} a_{\mathrm{L}}} \sqrt{\Omega}\right| \approx\left|\frac{\lambda_{\mathrm{R}}}{\lambda_{\mathrm{PIA}} a_{\mathrm{L}}}+\frac{\lambda_{\mathrm{I}}}{\hbar v_{F}}\right| .
$$

It depends on the relative signs of the entering SOC parameters whether this crossing appears for the valence $(n=-1)$ or conduction bands $(n=+1)$, see Fig. 7. However, for realistic graphenelike values of orbital and SOC parameters $k_{\mathrm{cr}} \approx 1 / a_{\mathrm{L}}$, what is far away from the Dirac valleys, and beyond the applicability of linear approximation.

Let us conclude by comparing the spin-orbit characteristics of systems with $C_{6 v}$ and $D_{3 d}$ point group symmetries. Both of them host principal-plane mirror asymmetry induced SOC coupling $\lambda_{\text {PIA }}$, but only $D_{3 d}$ simultaneously contain spaceinversion symmetry $\mathcal{I}$, see Fig. 3 . This seems to be crucial for answering the question whether PIA SOC causes spin splitting. It does in the absence of the space inversion- $C_{6 v}$ case, Eq. (64) - however, it does not in $D_{3 d}$ case, Eq. (48).

\section{4. $C_{3 v}$ case: sublattice resolved $\lambda_{\mathrm{I}}$ 's and $\lambda_{\mathrm{PIA}}$ 's couplings, and $\lambda_{\mathrm{R}}$}

The point group $C_{3 v}=\left\{E, 2 C_{3}^{\hat{z}}, 3 \Sigma_{v}\right\}$ is a subgroup of all three structural groups we have discussed earlier. For example, compared to the previous $C_{6 v}$ case, the point group $C_{3 v}$ lacks all the symmetries interchanging the sublattices. Hence the translationally invariant SOC Hamiltonian based on $\pi$ orbitals with $C_{3 v}$ and time reversal symmetries can be derived from the Hamiltonian $\mathcal{H}_{C_{6 v}}$, Eq. (59), making the next-nearest-neighbor SOC hoppings $i \lambda_{\mathrm{I}}$ and $\lambda_{\text {PIA }}$ sublattice dependent, i.e., $i \lambda_{\mathrm{I}} \rightarrow$ $\left\{i \lambda_{\mathrm{I}}^{\mathrm{A}}, i \lambda_{\mathrm{I}}^{\mathrm{B}}\right\}$ and $\lambda_{\mathrm{PIA}} \rightarrow\left\{\lambda_{\mathrm{PIA}}^{\mathrm{A}}, \lambda_{\mathrm{PIA}}^{\mathrm{B}}\right\}:$

$$
\begin{aligned}
\mathcal{H}_{C_{3 v}}= & \frac{i \lambda_{\mathrm{I}}^{\mathrm{A}}}{3 \sqrt{3}} \sum_{\sigma} \sum_{\langle m, n\rangle\rangle} v_{m, n}\left[\hat{s}_{z}\right]_{\sigma \sigma}\left|A_{m} \sigma\right\rangle\left\langle A_{n} \sigma\left|+\frac{i \lambda_{\mathrm{I}}^{\mathrm{B}}}{3 \sqrt{3}} \sum_{\sigma} \sum_{\langle m, n\rangle} v_{m, n}\left[\hat{s}_{z}\right]_{\sigma \sigma}\right| B_{m} \sigma\right\rangle\left\langle B_{n} \sigma\right| \\
& +\frac{2 \lambda_{\mathrm{PIA}}^{\mathrm{A}}}{3} \sum_{\sigma \neq \sigma^{\prime}} \sum_{\langle m, n\rangle\rangle}\left[i \hat{\boldsymbol{s}} \times \mathbf{d}_{m, n}\right]_{\sigma \sigma^{\prime}}\left|A_{m} \sigma\right\rangle\left\langle A_{n} \sigma^{\prime}\left|+\frac{2 \lambda_{\mathrm{PIA}}^{\mathrm{B}}}{3} \sum_{\sigma \neq \sigma^{\prime}} \sum_{\langle m, n\rangle\rangle}\left[i \hat{\boldsymbol{s}} \times \mathbf{d}_{m, n}\right]_{\sigma \sigma^{\prime}}\right| B_{m} \sigma\right\rangle\left\langle B_{n} \sigma^{\prime}\right| \\
& +\frac{2 i \lambda_{\mathrm{R}}}{3} \sum_{\sigma \neq \sigma^{\prime}} \sum_{\langle m, n\rangle}\left[\hat{\boldsymbol{s}} \times \mathbf{d}_{m, n}\right]_{\sigma \sigma^{\prime}}\left|X_{m} \sigma\right\rangle\left\langle X_{n} \sigma^{\prime}\right| .
\end{aligned}
$$

This Hamiltonian governs SOC effects in systems with broken sublattice symmetry (an effective staggered potential) and the fixed transverse direction (substrate or transverse electric field). Examples of such systems are semihydrogenated graphene [34] (graphone), graphene/TMDC heterostructures [52,53], silicene on the substrate etc. For the visualization, see Fig. 8.

The Bloch form of the Hamiltonian $\mathcal{H}_{C_{3 v}}$, Eq. (68), is straightforward since all the structural functions- $f_{\mathrm{I}}(\mathbf{q}), f_{\mathrm{P}}(\mathbf{q})$, $f_{\mathrm{R}}(\mathbf{q})$-were already given. Instead of that we fix the order of the Bloch basis $\left\{\left|A_{\mathbf{q}} \uparrow\right\rangle,\left|A_{\mathbf{q}} \downarrow\right\rangle,\left|B_{\mathbf{q}} \uparrow\right\rangle,\left|B_{\mathbf{q}} \downarrow\right\rangle\right\}$ and provide the low-energy Hamiltonian around $\mathbf{q}=\tau \mathbf{K}+\mathbf{k}$, including the orbital term with the staggered potential, $\mathcal{H}_{\text {orb }}(\tau \mathbf{K}+\mathbf{k})=$ $\hbar v_{F}\left(\tau k_{x} \hat{\sigma}_{x}-k_{y} \hat{\sigma}_{y}\right) \hat{s}_{0}+\Delta \hat{\sigma}_{z} \hat{s}_{0}$, in the matrix form

$$
\mathcal{H}_{\mathrm{eff}}(\tau \mathbf{K}+\mathbf{k})=\left(\begin{array}{cccc}
\tau \lambda_{\mathrm{I}}^{\mathrm{A}}+\Delta & -\lambda_{\mathrm{PIA}}^{\mathrm{A}}\left(i k_{x}+k_{y}\right) a_{\mathrm{L}} & \hbar v_{F}\left(\tau k_{x}+i k_{y}\right) & 2 i \lambda_{\mathrm{R}} \delta_{\tau \mathbf{K},+\mathbf{K}} \\
-\lambda_{\mathrm{PIA}}^{\mathrm{A}}\left(-i k_{x}+k_{y}\right) a_{\mathrm{L}} & -\tau \lambda_{\mathrm{I}}^{\mathrm{A}}+\Delta & 2 i \lambda_{\mathrm{R}} \delta_{\tau \mathbf{K},-\mathbf{K}} & \hbar v_{F}\left(\tau k_{x}+i k_{y}\right) \\
\hbar v_{F}\left(\tau k_{x}-i k_{y}\right) & -2 i \lambda_{\mathrm{R}} \delta_{\tau \mathbf{K},-\mathbf{K}} & -\tau \lambda_{\mathrm{I}}^{\mathrm{B}}-\Delta & -\lambda_{\mathrm{PIA}}^{\mathrm{B}}\left(i k_{x}+k_{y}\right) a_{\mathrm{L}} \\
-2 i \lambda_{\mathrm{R}} \delta_{\tau \mathbf{K},+\mathbf{K}} & \hbar v_{F}\left(\tau k_{x}-i k_{y}\right) & -\lambda_{\mathrm{PIA}}^{\mathrm{B}}\left(-i k_{x}+k_{y}\right) a_{\mathrm{L}} & \tau \lambda_{\mathrm{I}}^{\mathrm{B}}-\Delta
\end{array}\right) .
$$




\section{SYSTEMS IN ABSENCE OF TRANSLATIONAL INVARIANCE-IMPURITY INDUCED SOC HAMILTONIANS}

In the forthcoming sections, we discuss effective SOC Hamiltonians for hexagonal systems in the presence of locally chemisorbed impurities focusing on light adatoms and simple admolecules. The case of physisorbed heavy adelements is discussed in Refs. [40,58]. Since translational invariance is lost, the invariant expansion and decomposition into the irreps at high-symmetry points in the Brillouin zone are not applicable. However, the tight-binding-like methodology based on the local atomic orbitals and their group symmetry properties allows us to treat this problem very naturally. We assume a dilute coverage by light adsorbates and hence it is enough to investigate local SOC effects due to a single chemisorbed impurity-cluster formation and interference SOC effects among nearby impurity centers are therefore not discussed.

The electronic structure of an adatom and host (in most cases graphene) and the underlying molecular dynamics determine mainly three stable binding positions: the hollow, top, and bridge one. Equivalently, we can distinguish those adatom configurations through their local point group symmetries: $C_{6 v}$ for the hollow, $C_{3 v}$ for the top, and $C_{2 v}$ for the bridge one. For simplicity, we treat the chemisorbed adelement as monovalent, i.e., it bonds via a single effective orbital that is invariant under the local point-group symmetries. This monovalency assumption seems to be crude, though experience shows that the effective single-orbital description works very well [34-37]. However, an extension to the multiorbital case is technically straightforward.

As already stated, we are interested in local effective SOC Hamiltonians in the presence of an impurity, that are invariant under the corresponding local point group symmetries. Those can be then added to the global translational invariant Hamiltonians of the host systems as discussed in the previous sections. Locality for us means hoppings up to the next-nearest neighbors with respect to the adsorbed element. In what follows, we will label the adatom by $O$ and the corresponding atomic orbital by $|O\rangle$. Similarly, the adatom nearest-neighbor sites and orbitals will be denoted by $Y_{j}$ and $\left|Y_{j}\right\rangle$, respectively, and the next-nearest ones by $Z_{j}$ and $\left|Z_{j}\right\rangle$. The number of nearest and next-nearest carbon neighbors may vary depending on the adsorption configuration - this is indicated by the subscript $j$.

From the orbital point of view the minimal tight-binding description of the adatom that chemisorbs with its nearest neighbors is given by the Hamiltonian [25,34-37,89] $\mathcal{H}_{\text {orb }}$, which is defined as

$$
\begin{aligned}
\mathcal{H}_{\mathrm{orb}}= & \omega \sum_{\sigma} \sum_{\left\langle O, Y_{j}\right\rangle}|O \sigma\rangle\left\langle Y_{j} \sigma|+| Y_{j} \sigma\right\rangle\langle O \sigma| \\
& +\varepsilon \sum_{\sigma}|O \sigma\rangle\langle O \sigma| .
\end{aligned}
$$

The first term describes a hybridization $\omega$ between the adelement and its nearest neighbors (summation over $\left\langle O, Y_{j}\right\rangle$ ) and the second represents the adatom's on-site energy. For the remaining orbitals, we assume in the minimal-model scenario zero on-site contributions. The above orbital Hamiltonian is applicable to the hollow, top, and bridge configu-
TABLE II. Orbital tight-binding parameters for different adelements: hydrogen, fluorine, methyl, and copper in top and bridge position [34-37].

\begin{tabular}{lcr}
\hline \hline Adelement/model parameters (eV) & $\omega$ & \multicolumn{1}{c}{$\varepsilon$} \\
\hline Hydrogen (top) [34] & 7.50 & 0.16 \\
Fluorine (top) [35] & 5.50 & -2.20 \\
Methyl (top) [36] & 7.60 & -0.19 \\
Copper (top) [37] & 0.81 & 0.08 \\
Copper (bridge) [37] & 0.54 & 0.02 \\
\hline \hline
\end{tabular}

ration, respectively. The representative values of the orbital model parameters for different adelements are summarized in Table II.

\section{A. Adatom in hollow position}

$A b$ initio studies are unveiling that light metallic adatoms [90] from groups I-III and also heavy transition metals $[40,90,91]$ favor to adsorb above the centers of graphene hexagons, i.e., at the hollow positions. The same is true for light admolecules like $\mathrm{NH}_{3}, \mathrm{H}_{2} \mathrm{O}$, and $\mathrm{NO}_{2}$ [92]. The situation is schematically shown in Fig. 9. The central adelement $O$ has six nearest carbon neighbors $Y_{j}$ and since the out-of plane position of the adatom fixes the orientation of the perpendicular $z$ axis the structure is locally described by the point group $C_{6 v}$. We will focus on a SOC Hamiltonian including the adatom orbital $|O\rangle$ and the $\pi$-state carbon orbitals $\left|Y_{j}\right\rangle$ of its direct nearest neighbors only. We first discuss the SOC mediated hoppings among $Y$ 's sites and then we account for hoppings between the adatom orbital $|O\rangle$ and its six neighboring orbitals $\left|Y_{j}\right\rangle$. Since the translational symmetry is lost, we avoid using attributes like intrinsic, Rashba, and so on for the local SOC mediated hoppings. Instead we use the full taxonomy: spin-conserving (next) nearest-neighbor hopping $\Lambda_{\mathrm{c}}^{(\mathrm{n}) \mathrm{n}}$ and spin-flipping (next) nearest-neighbor hopping $\Lambda_{\mathrm{f}}^{(\mathrm{n}) \mathrm{n}}$, respectively, reserving for the local SOC capital $\Lambda$.

The translationally invariant SOC Hamiltonian with $C_{6 v}$ symmetry was discussed in the preceding section, Eq. (59). Making it local, the global terms- $i \lambda_{\mathrm{I}}, \lambda_{\mathrm{PIA}}$, and $i \lambda_{\mathrm{R}}-$ can not diminish. They would be respectively recast into their local analogs $-i \Lambda_{\mathrm{c}}^{\mathrm{nn}}, \Lambda_{\mathrm{f}}^{\mathrm{nn}}$, and $i \Lambda_{\mathrm{f}}^{\mathrm{n}}$. Since all the $Y$ 's sites are equivalent, there are not sublattice resolved partners of those $\Lambda$ 's. In addition, the lack of the translational invariance allows now also the purely imaginary spin-conserving nearest-neighbor hopping $i \Lambda_{\mathrm{c}}^{\mathrm{n}} \simeq\left\langle Y_{i} \sigma\left|\hat{H}_{\mathrm{so}}\right| Y_{i+1} \sigma\right\rangle$; see no-go arguments of Sec. III B. So finally, there are four independent

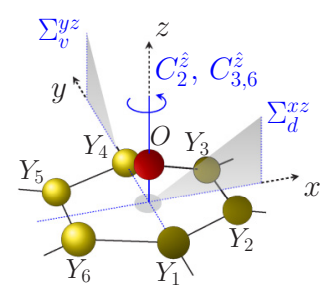

FIG. 9. Adatom bonded in the hollow position: local point group symmetry $C_{6 v}$-similar to graphene in the transverse external electric field, atom labeling convention, axes orientations, and $C_{6 v}$ group operations as discussed in the text. 
SOC mediated hoppings among the $Y$ 's sites, which-in analogy with the former analysis - can be defined as follows:

$$
\begin{aligned}
i \Lambda_{\mathrm{c}}^{\mathrm{nn}} & =\left\langle Y_{5} \uparrow\left|\hat{H}_{\mathrm{so}}\right| Y_{3} \uparrow\right\rangle, \\
\Lambda_{\mathrm{f}}^{\mathrm{nn}} & =\left\langle Y_{5} \uparrow\left|\hat{H}_{\mathrm{so}}\right| Y_{3} \downarrow\right\rangle, \\
i \Lambda_{\mathrm{f}}^{\mathrm{n}} & =\left\langle Y_{3} \uparrow\left|\hat{H}_{\mathrm{so}}\right| Y_{2} \downarrow\right\rangle, \\
i \Lambda_{\mathrm{c}}^{\mathrm{n}} & =\left\langle Y_{2} \uparrow\left|\hat{H}_{\mathrm{so}}\right| Y_{3} \uparrow\right\rangle ;
\end{aligned}
$$

for the labeling of atomic sites see Fig. 9. Here we no longer use the numerical prefactors $1 / 3 \sqrt{3}$ and $2 / 3$, which were convenient for the low-energy $k$-space expansions. The SOC mediated hoppings among the $Y$ sites at different configurations can be obtained by Eqs. (5), (14), and (56). For $i \Lambda_{\mathrm{c}}^{\mathrm{n}}$, we have in analogy with Eq. (14) the following identity which holds for any two nearest neighbors $Y_{j}$ and $Y_{k}$ of the adatom $O$ :

$$
\left\langle Y_{j} \sigma\left|\hat{H}_{\mathrm{so}}\right| Y_{k} \sigma\right\rangle=\tilde{v}_{Y_{j}, Y_{k}}\left[\hat{s}_{z}\right]_{\sigma \sigma} i \Lambda_{\mathrm{c}}^{\mathrm{n}} .
$$

Here, $\tilde{v}_{Y_{j}, Y_{k}}=+1(-1)$ if the hopping from the site $Y_{k}$ to $Y_{j}$ via a central adatom $O$ is counter clockwise (clockwise).

Next, we examine SOC mediated hoppings between the adatom orbital $|O\rangle$ and its neighbors $\left|Y_{j}\right\rangle$ along the hexagonal ring. For that it is enough to look at matrix elements $\left\langle O \uparrow\left|\hat{H}_{\mathrm{so}}\right| Y_{1} \uparrow\right\rangle$ and $\left\langle O \uparrow\left|\hat{H}_{\mathrm{so}}\right| Y_{1} \downarrow\right\rangle$, respectively. Assuming $|O\rangle$ is $C_{6 v}$ and time reversal invariant-i.e., $\mathcal{S}|O\rangle=|O\rangle$ for any $\mathcal{S} \in C_{6 v}$ and $\mathcal{T}|O\rangle=|O\rangle$ as would be the case of alkali metals - we can show that the first of the above matrix elements is identically zero and the second is purely imaginary. Particulary,

$$
\begin{aligned}
\left\langle O \uparrow\left|\hat{H}_{\mathrm{so}}\right| Y_{1} \uparrow\right\rangle & \stackrel{(1 c)}{=}\left\langle-i \Sigma_{v}^{y z}[O \downarrow]\left|\hat{H}_{\mathrm{so}}\right|-i \Sigma_{v}^{y z}\left[Y_{1} \downarrow\right]\right\rangle \\
& \stackrel{(3)}{=}\left\langle O \downarrow\left|\hat{H}_{\mathrm{so}}\right| Y_{1} \downarrow\right\rangle \stackrel{(7)}{=}-\left\langle O \uparrow\left|\hat{H}_{\mathrm{so}}\right| Y_{1} \uparrow\right\rangle .
\end{aligned}
$$

For the spin-flip hopping, we get

$$
\begin{aligned}
\left\langle O \uparrow\left|\hat{H}_{\mathrm{so}}\right| Y_{1} \downarrow\right\rangle & \stackrel{(1 c)}{=}\left\langle-i \Sigma_{v}^{y z}[O \downarrow]\left|\hat{H}_{\mathrm{so}}\right|-i \Sigma_{v}^{y z}\left[Y_{1} \uparrow\right]\right\rangle \\
& \stackrel{(3)}{=}\left\langle O \downarrow\left|\hat{H}_{\mathrm{so}}\right| Y_{1} \uparrow\right\rangle \stackrel{(5)}{=}-\overline{\left\langle O \uparrow\left|\hat{H}_{\mathrm{so}}\right| Y_{1} \downarrow\right\rangle}
\end{aligned}
$$

what allows us to define the SOC term,

$$
i \Lambda_{\mathrm{f}}^{\mathrm{On}}=\left\langle O \uparrow\left|\hat{H}_{\mathrm{so}}\right| Y_{1 \downarrow} \downarrow .\right.
$$

Equivalent couplings can be specified by reflections, rotations and time reversal, e.g., by applying $\mathcal{R}_{\frac{\pi}{3}}^{\hat{z}}$, we get

$$
\begin{aligned}
\left\langle O \uparrow\left|\hat{H}_{\mathrm{So}}\right| Y_{2} \downarrow\right\rangle & =\left\langle e^{i \frac{\pi}{6}} \mathcal{R}_{\frac{\pi}{3}}^{\hat{z}}[O \uparrow]\left|\hat{H}_{\mathrm{so}}\right| e^{-i \frac{\pi}{6}} \mathcal{R}_{\frac{\pi}{3}}^{\hat{z}}\left[Y_{1} \downarrow\right]\right\rangle \\
& \stackrel{(3)}{=} e^{-i \frac{\pi}{3}}\left\langle O \uparrow\left|\hat{H}_{\mathrm{so}}\right| Y_{1} \downarrow\right\rangle \stackrel{(71)}{=} e^{-i \frac{\pi}{3}} i \Lambda_{\mathrm{f}}^{\text {On }},
\end{aligned}
$$

and in general, for any $Y_{j}$ and $\sigma \neq \sigma^{\prime}$, we have

$$
\left\langle O \sigma\left|\hat{H}_{\mathrm{so}}\right| Y_{j} \sigma^{\prime}\right\rangle=\left[\hat{\boldsymbol{s}} \times \mathbf{d}_{O, Y_{j}}\right]_{\sigma \sigma^{\prime}} i \Lambda_{\mathrm{f}}^{\mathrm{On}},
$$

where the meaning of $\mathbf{d}_{O, Y_{j}}$ is identical as before-a unit vector in $x y$ plane pointing from site $Y_{j}$ to $O$. The local SOC Hamiltonian for the hollow position possesses five SOC terms- $i \Lambda_{\mathrm{c}}^{\mathrm{n}}, i \Lambda_{\mathrm{c}}^{\mathrm{nn}}, i \Lambda_{\mathrm{f}}^{\mathrm{n}}, \Lambda_{\mathrm{f}}^{\mathrm{nn}}$, and $i \Lambda_{\mathrm{f}}^{\text {On }}$-and is given as follows:

$$
\begin{aligned}
\mathcal{H}_{\mathrm{so}}^{\mathrm{hol}}= & i \Lambda_{\mathrm{c}}^{\mathrm{n}} \sum_{\sigma} \sum_{\left\langle Y_{j}, Y_{k}\right\rangle} \tilde{v}_{Y_{j}, Y_{k}}\left[\hat{s}_{z}\right]_{\sigma \sigma}\left|Y_{j} \sigma\right\rangle\left\langle Y_{k} \sigma\right| \\
& +i \Lambda_{\mathrm{c}}^{\mathrm{nn}} \sum_{\sigma} \sum_{\left\langle\left\langle Y_{j}, Y_{k}\right\rangle\right\rangle} v_{Y_{j}, Y_{k}}\left[\hat{s}_{z}\right]_{\sigma \sigma}\left|Y_{j} \sigma\right\rangle\left\langle Y_{k} \sigma\right| \\
& +i \Lambda_{\mathrm{f}}^{\mathrm{n}} \sum_{\sigma \neq \sigma^{\prime}} \sum_{\left\langle Y_{j}, Y_{k}\right\rangle}\left[\hat{\boldsymbol{s}} \times \mathbf{d}_{Y_{j}, Y_{k}}\right]_{\sigma \sigma^{\prime}}\left|Y_{j} \sigma\right\rangle\left\langle Y_{k} \sigma^{\prime}\right| \\
& +\Lambda_{\mathrm{f}}^{\mathrm{nn}} \sum_{\sigma \neq \sigma^{\prime}} \sum_{\left\langle Y_{j}, Y_{k}\right\rangle}\left[i \hat{\boldsymbol{s}} \times \mathbf{d}_{Y_{j}, Y_{k}}\right]_{\sigma \sigma^{\prime}}\left|Y_{j} \sigma\right\rangle\left\langle Y_{k} \sigma^{\prime}\right| \\
& +i \Lambda_{\mathrm{f}}^{\mathrm{On}} \sum_{\sigma \neq \sigma^{\prime}} \sum_{\left\langle O, Y_{j}\right\rangle}\left[\hat{\boldsymbol{s}} \times \mathbf{d}_{O, Y_{j}}\right]_{\sigma \sigma^{\prime}}|O \sigma\rangle\left\langle Y_{j} \sigma^{\prime}\right|+\text { H.c.. }
\end{aligned}
$$

Again, the summation over the nearest- and next-nearest neighbors is specified by $\langle$,$\rangle and \langle\langle\rangle$,$\rangle brackets, respectively;$ for the atomic configurations that enter $v$, $\tilde{v}$, and d see Fig. 9.

\section{B. Adatom in top position}

Adsorption in the top position seems to be favorable for light atoms like hydrogen [34,93], fluorine [35,94,95] and copper $[37,96,97]$, the heavier gold atom [90,97], and, for example, also the light methyl admolecule [36]. The model configuration has a local $C_{3 v}$ point group symmetry and is displayed in Fig. 10 - an adatom $O$ binding on the top possesses one nearest $Y$ neighbor, three second-nearest $Z$ neighbors, and six thirdnearest $W$ neighbors. To compare the global and local $C_{3 v}$ cases, which have different centers of symmetry, we consider also mutual SOC hoppings implementing the third-nearest $W$ neighbors.

The local $C_{3 v}$-invariant SOC Hamiltonian accounting for SOC mediated hoppings among the $Y, Z$, and $W$ carbon sites-the SOC hoppings connecting the adatom will be discussed later-can be naturally derived from the global $C_{3 v}$ Hamiltonian, Eq. (68). In analogy with the global $i \lambda_{\mathrm{I}}^{\mathrm{A}}, i \lambda_{\mathrm{I}}^{\mathrm{B}}$, $\lambda_{\text {PIA }}^{\mathrm{A}}, \lambda_{\mathrm{PIA}}^{\mathrm{B}}$, and $i \lambda_{\mathrm{R}}$ couplings, we correspondingly have

$$
\begin{aligned}
i \Lambda_{\mathrm{c}}^{\mathrm{YW}} & =\left\langle Y \uparrow\left|\hat{H}_{\mathrm{so}}\right| W_{3} \uparrow\right\rangle, \\
i \Lambda_{\mathrm{c}}^{\mathrm{ZZ}} & =\left\langle Z_{3} \uparrow\left|\hat{H}_{\mathrm{so}}\right| Z_{2} \uparrow\right\rangle, \\
\Lambda_{\mathrm{f}}^{\mathrm{YW}} & =\left\langle Y \uparrow\left|\hat{H}_{\mathrm{so}}\right| W_{3} \downarrow\right\rangle,
\end{aligned}
$$

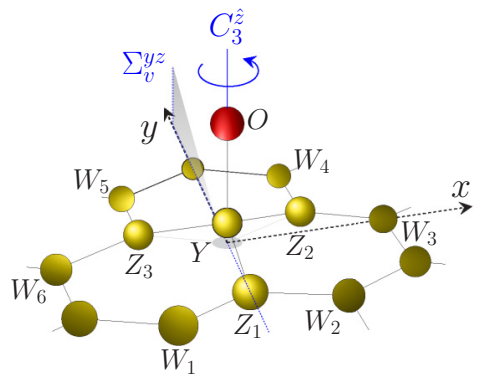

FIG. 10. Adatom bonded in the top position with a center of symmetry on the bonding axis: the local point group symmetry $C_{3 v}$, atom labeling convention, axes orientations, and $C_{3 v}$ group operations as discussed in the main text. 


$$
\begin{aligned}
\Lambda_{\mathrm{f}}^{\mathrm{ZZ}} & =\left\langle Z_{3} \uparrow\left|\hat{H}_{\mathrm{so}}\right| Z_{2} \downarrow\right\rangle, \\
i \Lambda_{\mathrm{f}}^{\mathrm{YZ}} & =\left\langle Y \uparrow\left|\hat{H}_{\mathrm{so}}\right| Z_{1} \downarrow\right\rangle .
\end{aligned}
$$

Here, again the subscripts "c" and " $\mathrm{f}$ " stand for spinconserving and spin-flipping hoppings, respectively, and the superscripts made from $Y, Z$, and $W$ encode particular nearestor next-nearest-neighbor hoppings among the $Y, Z$, and $W$ carbon sites. For atomic configuration and labeling see Fig. 10.

Using Eq. (9), we see that $i \Lambda_{\mathrm{c}}^{\mathrm{YW}}$ and $i \Lambda_{\mathrm{c}}^{\mathrm{ZZ}}$-local analogs of $i \lambda_{\mathrm{I}}^{\mathrm{A}}$ and $i \lambda_{\mathrm{I}}^{\mathrm{B}}$-are purely imaginary. Similarly, substituting in Eq. (35) $A_{2}$ by $Z_{2}$ and $A_{3}$ by $Z_{3}$ unveils that $\Lambda_{\mathrm{f}}^{\mathrm{ZZ}}$-a local analog of $\lambda_{\mathrm{PIA}}^{\mathrm{B}}$-is purely real. Readapting the argumentation used in Eq. (55) to the situation displayed at Fig. 10, we get

$$
\begin{aligned}
\left\langle Y \uparrow\left|\hat{H}_{\mathrm{so}}\right| Z_{1} \downarrow\right\rangle & =\left\langle-i \Sigma_{v}^{y z}[Y \downarrow]\left|\hat{H}_{\mathrm{so}}\right|-i \Sigma_{v}^{y z}\left[Z_{1} \uparrow\right]\right\rangle \\
& \stackrel{(3)}{=}\left\langle Y \downarrow\left|\hat{H}_{\mathrm{so}}\right| Z_{1} \uparrow\right\rangle \stackrel{(5)}{=}-\overline{\left\langle Y \uparrow\left|\hat{H}_{\mathrm{so}}\right| Z_{1} \downarrow\right\rangle},
\end{aligned}
$$

what confirms that $i \Lambda_{\mathrm{f}}^{\mathrm{YZ}}$-a local analog of $i \lambda_{\mathrm{R}}$-is purely imaginary.

What differs from the global $C_{3 v}$ case is the spin-flip coupling $\Lambda_{\mathrm{f}}^{\mathrm{YW}}$-an analog of $\lambda_{\mathrm{PIA}}^{\mathrm{A}}$. Since now the sites $Y$ and $W$ are not interchangeable we cannot use the argument analogous to Eq. (35) and hence $\Lambda_{\mathrm{f}}^{\mathrm{YW}}$ is in general complex. This slightly affects the former phase-factor formula, Eq. (58), which now read

$$
\begin{aligned}
\left\langle Y \sigma\left|\hat{H}_{\mathrm{so}}\right| W_{j} \sigma^{\prime}\right\rangle= & {\left[i \hat{\boldsymbol{s}} \times \mathbf{d}_{Y, W_{j}}\right]_{\sigma \sigma^{\prime}}\left[\operatorname{Re}\left(\Lambda_{\mathrm{f}}^{\mathrm{YW}}\right)\right.} \\
& \left.+i v_{Y, W_{j}} \operatorname{Im}\left(\Lambda_{\mathrm{f}}^{\mathrm{YW}}\right)\right] .
\end{aligned}
$$

The meaning of $\mathbf{d}_{Y, W_{j}}$ and $v_{Y, W_{j}}$ stays the same as before. The spin-orbit mediated hoppings among the carbon atoms in the vicinity of the impurity site $O$ are now recapped.

In what follows, we discuss the SOC mediated hoppings $\left\langle O \sigma\left|\hat{H}_{\mathrm{so}}\right| Y \sigma^{\prime}\right\rangle$ and $\left\langle O \sigma\left|\hat{H}_{\mathrm{so}}\right| Z_{i} \sigma^{\prime}\right\rangle$ that couple directly to the adatom orbital $|O\rangle$-assuming it is $C_{3 v}$ and time reversal invariant. Repeating the discussion at the end of previous section, see Eqs. (73) and (74), we immediately get

$$
\begin{aligned}
& i \Lambda_{\mathrm{c}}^{\mathrm{OZ}}=\left\langle O \uparrow\left|\hat{H}_{\mathrm{so}}\right| Z_{1} \uparrow\right\rangle \equiv 0, \\
& i \Lambda_{\mathrm{f}}^{\mathrm{OZ}}=\left\langle O \uparrow\left|\hat{H}_{\mathrm{so}}\right| Z_{1} \downarrow\right\rangle \neq 0 .
\end{aligned}
$$

To show that $\left\langle O \uparrow\left|\hat{H}_{\mathrm{so}}\right| Y \uparrow\right\rangle$ and $\left\langle O \uparrow\left|\hat{H}_{\mathrm{so}}\right| Y \downarrow\right\rangle$ are zero one can proceed as follows: for the first term, we have

$$
\begin{aligned}
\left\langle O \uparrow\left|\hat{H}_{\mathrm{so}}\right| Y \uparrow\right\rangle & \stackrel{(7)}{=}-\left\langle O \downarrow\left|\hat{H}_{\mathrm{so}}\right| Y \downarrow\right\rangle \\
& \stackrel{(1 c)}{=}-\left\langle-i \Sigma_{v}^{y z}[O \uparrow]\left|\hat{H}_{\mathrm{so}}\right|-i \Sigma_{v}^{y z}[Y \uparrow]\right\rangle \\
& \stackrel{(3)}{=}-\left\langle O \uparrow\left|\hat{H}_{\mathrm{so}}\right| Y \uparrow\right\rangle,
\end{aligned}
$$

which implies that $\left\langle O \uparrow\left|\hat{H}_{\mathrm{so}}\right| Y \uparrow\right\rangle \equiv 0$. To show that $\left\langle O \uparrow\left|\hat{H}_{\mathrm{so}}\right| Y \downarrow\right\rangle$ is zero, we apply rotations $\mathcal{R}_{ \pm \frac{2 \pi}{3}}^{\hat{z}} \in C_{3 v}$, then

$$
\begin{gathered}
\left\langle O \uparrow\left|\hat{H}_{\mathrm{so}}\right| Y \downarrow\right\rangle \stackrel{(1 a)}{=}\left\langle e^{ \pm i \frac{\pi}{3}} \mathcal{R}_{ \pm \frac{2 \pi}{3}}^{\hat{z}}[O \uparrow]\left|\hat{H}_{\mathrm{so}}\right| e^{\mp i \frac{\pi}{3}} \mathcal{R}_{ \pm \frac{2 \pi}{3}}^{\hat{z}}[Y \downarrow]\right\rangle \\
\stackrel{(3)}{=} e^{\mp i \frac{2 \pi}{3}}\left\langle O \uparrow\left|\hat{H}_{\mathrm{so}}\right| Y \downarrow\right\rangle .
\end{gathered}
$$

The above relation can be fulfilled only by zero, therefore $\left\langle O \uparrow\left|\hat{H}_{\mathrm{so}}\right| Y \downarrow\right\rangle=0$.
Summarizing Eqs. (79) and (83), we have in total six spinorbit couplings-four purely imaginary $i \Lambda_{\mathrm{c}}^{\mathrm{YW}}, i \Lambda_{\mathrm{c}}^{\mathrm{ZZ}}, i \Lambda_{\mathrm{f}}^{\mathrm{YZ}}$, and $i \Lambda_{\mathrm{f}}^{\mathrm{OZ}}$, one purely real $\Lambda_{\mathrm{fZ}}^{\mathrm{ZZ}}$, and one in general complex $\Lambda_{\mathrm{f}}^{\mathrm{YW}}$. The local SOC Hamiltonian with $C_{3 v}$ symmetry that corresponds to the impurity in the top position reads

$$
\begin{aligned}
\mathcal{H}_{\mathrm{so}}^{\mathrm{top}}= & i \Lambda_{\mathrm{c}}^{\mathrm{YW}} \sum_{\sigma} \sum_{\left\langle Y, W_{j}\right\rangle} v_{Y, W_{j}}\left[\hat{s}_{Z}\right]_{\sigma \sigma}|Y \sigma\rangle\left\langle W_{j} \sigma\right|+\text { H.c. } \\
& +i \Lambda_{\mathrm{c}}^{\mathrm{ZZ}} \sum_{\sigma} \sum_{\left\langle\left\langle Z_{j}, Z_{k}\right\rangle\right.} v_{Z_{j}, Z_{k}}\left[\hat{s}_{z}\right]_{\sigma \sigma}\left|Z_{j} \sigma\right\rangle\left\langle Z_{k} \sigma\right| \\
& +\sum_{\sigma \neq \sigma^{\prime}} \sum_{\left\langle Y, W_{j}\right\rangle}\left[i \hat{\boldsymbol{s}} \times \mathbf{d}_{Y, W_{j}}\right]_{\sigma \sigma^{\prime}}\left[\operatorname{Re}\left(\Lambda_{\mathrm{f}}^{\mathrm{YW}}\right)\right. \\
& \left.+i v_{Y, W_{j}} \operatorname{Im}\left(\Lambda_{\mathrm{f}}^{\mathrm{YW}}\right)\right]|Y \sigma\rangle\left\langle W_{j} \sigma^{\prime}\right|+\text { H.c. } \\
& +\Lambda_{\mathrm{f}}^{\mathrm{ZZ}} \sum_{\sigma \neq \sigma^{\prime}} \sum_{\left\langle Z_{j}, Z_{k}\right\rangle}\left[i \hat{\boldsymbol{s}} \times \mathbf{d}_{Z_{j}, Z_{k}}\right]_{\sigma \sigma^{\prime}}\left|Z_{j} \sigma\right\rangle\left\langle Z_{k} \sigma^{\prime}\right| \\
& +i \Lambda_{\mathrm{f}}^{\mathrm{YZ}} \sum_{\sigma \neq \sigma^{\prime}} \sum_{\left\langle Y, Z_{j}\right\rangle}\left[\hat{\boldsymbol{s}} \times \mathbf{d}_{Y, Z_{j}}\right]_{\sigma \sigma^{\prime}}|Y \sigma\rangle\left\langle Z_{j} \sigma^{\prime}\right|+\text { H.c. } \\
& +i \Lambda_{\mathrm{f}}^{\mathrm{OZ}} \sum_{\sigma \neq \sigma^{\prime}} \sum_{\left\langle O, Z_{j}\right\rangle}\left[\hat{\boldsymbol{s}} \times \mathbf{d}_{O, Z_{j}}\right]_{\sigma \sigma^{\prime}}|O \sigma\rangle\left\langle Z_{j} \sigma^{\prime}\right|+\text { H.c.. }
\end{aligned}
$$

\section{Adatom in bridge position}

Oxygen and nitrogen are theoretically predicted to bond in the bridge position [94]. However, also for impurities in the top position like copper $[37,96,97]$ and gold $[90,97]$ the energy difference between the top and bridge configurations is relatively small and therefore their bridge realization becomes quite probable. Similarly, the light admolecules like $\mathrm{CO}, \mathrm{NO}$ and $\mathrm{NO}_{2}$ prefer to adsorb [92] equally likely to the hollow and bridge positions. For those reasons, we discuss in this section an effective SOC Hamiltonian that works for light adelements in the bridge configuration. Particulary, by bridge we understand a configuration when the adatom $O$ splits a nearest-neighbor bond between two $-Y_{1}$ and $Y_{2}$-carbon sites, see Fig. 11. Such a structure possesses $C_{2 v}$ point group symmetry, which comprises two nonequivalent reflection planes $\Sigma_{d}^{x z}$ and $\Sigma_{v}^{y z}$, and $C_{2}$ rotation around the axis of their intersection; see Fig. 11. As the order of this group is lower compared to the above cases we expect more SOC mediated matrix elements which in general would be complex-valued. Even within the approximation that keeps only nearest and next-nearest-neighbor hoppings among $O, Y$, and $Z$ sites, the

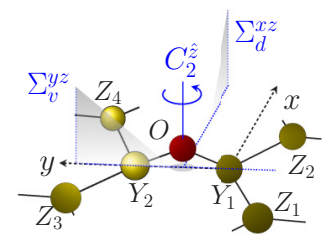

FIG. 11. Adatom bonded in the bridge position with a center of symmetry on the vertical axis passing the adatom: the local point group symmetry $C_{2 v}$, atom labeling convention, axes orientations, and $C_{2 v}$ group operations as discussed in the main text. 
effective SOC Hamiltonian contains eight hoppings. Three of them are spin-conserving (and hence purely imaginary) and the remaining five are spin-flipping,

$$
\begin{aligned}
i \Lambda_{\mathrm{c}}^{\mathrm{YZ}} & =\left\langle Y_{2} \uparrow\left|\hat{H}_{\mathrm{so}}\right| Z_{4} \uparrow\right\rangle, \\
i \Lambda_{\mathrm{c}}^{\mathrm{OZ}} & =\left\langle O \uparrow\left|\hat{H}_{\mathrm{so}}\right| Z_{4} \uparrow\right\rangle, \\
i \Lambda_{\mathrm{c}}^{\mathrm{ZZ}} & =\left\langle Z_{1} \uparrow\left|\hat{H}_{\mathrm{so}}\right| Z_{2} \uparrow\right\rangle, \\
i \Lambda_{\mathrm{f}}^{\mathrm{OY}} & =\left\langle O \uparrow\left|\hat{H}_{\mathrm{so}}\right| Y_{1} \downarrow\right\rangle, \\
i \Lambda_{\mathrm{f}}^{\mathrm{YY}} & =\left\langle Y_{1} \uparrow\left|\hat{H}_{\mathrm{so}}\right| Y_{2} \downarrow\right\rangle, \\
\operatorname{Re}\left(\Lambda_{\mathrm{f}}^{\mathrm{YZ}}\right)+\mathrm{i} \operatorname{Im}\left(\Lambda_{\mathrm{f}}^{\mathrm{YZ}}\right) & =\left\langle Y_{2} \uparrow\left|\hat{H}_{\mathrm{so}}\right| Z_{4} \downarrow\right\rangle, \\
\operatorname{Re}\left(\Lambda_{\mathrm{f}}^{\mathrm{OZ}}\right)+\mathrm{i} \operatorname{Im}\left(\Lambda_{\mathrm{f}}^{\mathrm{OZ}}\right) & =\left\langle O \uparrow\left|\hat{H}_{\mathrm{so}}\right| Z_{4} \downarrow\right\rangle, \\
\Lambda_{\mathrm{f}}^{\mathrm{ZZ}} & =\left\langle Z_{2} \uparrow\left|\hat{H}_{\mathrm{so}}\right| Z_{1} \downarrow\right\rangle .
\end{aligned}
$$

Let us shortly comment on three of the above emerging couplings-Eqs. (87a), (87f), and (87g). The absence of the translational invariance allows spin-conserving hopping $i \Lambda_{\mathrm{c}}^{\mathrm{YZ}}$ between the nearest-neighbor sites $Y$ and $Z$-similar coupling was encountered in the local $C_{6 v}$ case for the adatom in hollow position. For the same reason, there are the spin-flip hoppings $\Lambda_{\mathrm{f}}^{\mathrm{YZ}}$ —among the nearest neighbors-

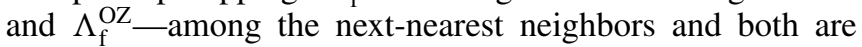
complex-valued in general.

Altogether, we can write the local Hamiltonian for the local $C_{2 v}$ symmetric structure in a closed form, with the help of the definitions that we introduced above, as follows:

$$
\begin{aligned}
& \mathcal{H}_{\mathrm{so}}^{\text {brid }}=i \Lambda_{\mathrm{c}}^{\mathrm{YZ}} \sum_{\sigma} \sum_{\left\langle Y_{j}, Z_{k}\right\rangle} v_{O, Z_{k}}\left[\hat{s}_{z}\right]_{\sigma \sigma}\left|Y_{j} \sigma\right\rangle\left\langle Z_{k} \sigma\right|+\text { H.c. } \\
& +i \Lambda_{\mathrm{c}}^{\mathrm{OZ}} \sum_{\sigma} \sum_{\left\langle\left\langle O, Z_{k}\right\rangle\right.} v_{O, Z_{k}}\left[\hat{s}_{z}\right]_{\sigma \sigma}|O \sigma\rangle\left\langle Z_{k} \sigma\right|+\text { H.c. } \\
& +i \Lambda_{\mathrm{c}}^{\mathrm{ZZ}} \sum_{\sigma} \sum_{\left\langle\left\langle Z_{j}, Z_{k}\right\rangle\right\rangle} v_{Z_{j}, Z_{k}}\left[\hat{s}_{z}\right]_{\sigma \sigma}\left|Z_{j} \sigma\right\rangle\left\langle Z_{k} \sigma\right| \\
& +i \Lambda_{\mathrm{f}}^{\mathrm{OY}} \sum_{\sigma \neq \sigma^{\prime}} \sum_{\left\langle O, Y_{j}\right\rangle}\left[\hat{\boldsymbol{s}} \times \mathbf{d}_{O, Y_{j}}\right]_{\sigma \sigma^{\prime}}|O \sigma\rangle\left\langle Y_{j} \sigma^{\prime}\right|+\text { H.c. } \\
& +i \Lambda_{\mathrm{f}}^{\mathrm{YY}} \sum_{\sigma \neq \sigma^{\prime}}\left[\hat{\boldsymbol{s}} \times \mathbf{d}_{Y_{1}, Y_{2}}\right]_{\sigma \sigma^{\prime}}\left|Y_{1} \sigma\right\rangle\left\langle Y_{2} \sigma^{\prime}\right|+\text { H.c. } \\
& +\sum_{\sigma \neq \sigma^{\prime}} \sum_{\left\langle Y_{j}, Z_{k}\right\rangle}\left[v_{O, Z_{k}}\left[i \hat{s}_{y}\right]_{\sigma \sigma^{\prime}} \operatorname{Re}\left(\Lambda_{\mathrm{f}}^{\mathrm{YZ}}\right)+i \operatorname{Im}\left(\Lambda_{\mathrm{f}}^{\mathrm{YZ}}\right)\right] \\
& \times \operatorname{sgn}\left[\mathbf{d}_{O, Y_{j}} \cdot \mathbf{d}_{Y_{1}, Y_{2}}\right]\left|Y_{j} \sigma\right\rangle\left\langle Z_{k} \sigma^{\prime}\right|+\text { H.c. } \\
& +\sum_{\sigma \neq \sigma^{\prime}} \sum_{\left\langle\left\langle, Z_{j}\right\rangle\right\rangle}\left[v_{O, Z_{j}}\left[i \hat{s}_{y}\right]_{\sigma \sigma^{\prime}} \operatorname{Re}\left(\Lambda_{\mathrm{f}}^{\mathrm{OZ}}\right)+i \operatorname{Im}\left(\Lambda_{\mathrm{f}}^{\mathrm{OZ}}\right)\right] \\
& \times \operatorname{sgn}\left[\mathbf{d}_{O, Z_{j}} \cdot \mathbf{d}_{Y_{1}, Y_{2}}\right]|O \sigma\rangle\left\langle Z_{j} \sigma^{\prime}\right|+\text { H.c. } \\
& +\Lambda_{\mathrm{f}}^{\mathrm{ZZ}} \sum_{\sigma \neq \sigma^{\prime}} \sum_{\left\langle Z_{j}, Z_{k}\right\rangle}\left[i \hat{\boldsymbol{s}} \times \mathbf{d}_{Z_{j}, Z_{k}}\right]_{\sigma \sigma^{\prime}}\left|Z_{j} \sigma\right\rangle\left\langle Z_{k} \sigma^{\prime}\right| \text {. }
\end{aligned}
$$

In the first and sixth lines, in which we are summing over the nearest neighbors $\left\langle Y_{j}, Z_{k}\right\rangle$, the symbol $v_{O, Z_{k}}$ has the following meaning; it equals $1(-1)$ if the path $Z_{k} \rightarrow Y_{j}$ after extension to the next-nearest-neighbor path $Z_{k} \rightarrow Y_{j} \rightarrow O$ becomes counter clockwise (clockwise).

\section{FINAL REMARKS AND CONCLUSION}

As already noted, the lower the symmetry, the more SOC parameters enter the effective model Hamiltonians. Before applying a particular model to spin-transport studies, two issues should be resolved. First, figure out the realistic strengths of SOC parameters and, second, reduce the number of the parameters as much as possible. For that, one should employ first-principles calculations together with physical intuition and common sense.

To describe our strategy, we start with ab initio calculations considering a large graphene supercell with one adelement bonded in a given configuration. The larger the supercell, the weaker are the interactions among the periodic images, and the more representative the dilute coverage limit is realized. Analyzing local DOS and its atomic orbital decomposition, we directly test whether the system can be properly described by the adequate Hamiltonian model, i.e., carbon $\pi$ orbitals and an effective adatom level. In all the cases yet analyzed-hydrogen [34], fluorine [35], $\mathrm{CH}_{3}$ group [36], and copper [37] (both in top and bridge configurations) - the effective models with effective adatom orbitals work perfectly. For example, for fluorine [35] the remaining $2 p$ orbitals contribute to electronic bands far below the Fermi level, and hence the low-energy physics is dominated by $2 p_{z}$ orbital. Fitting the spin-orbit induced band splittings would give us the strengths of the sought SOC parameters. The aim is to find a minimal set of best-fitting parameters to keep the model simple and simultaneously capture the main features in SOC induced band splittings. It might not be necessary to take into account all the symmetry-allowed coupling parameters. For that some intuition, experience and an input from the DFT are helpful, e.g., the possibility to turn off in first-principles calculations SOC interaction on the adatom, or shift away the Fermi level Bloch states composed from the (un)wanted atomic orbitals [98]. All that helps to trace the importance and interpretation of the effective spin-orbit couplings. Table III summarizes the relevant spin-orbit couplings including their strengths as taken from Refs. [34-37]. The general tendency is obvious, the heavier the adelement, the stronger are the local SOC parameters. Comparing their strengths with respect to the graphene intrinsic SOC, we see that hydrogen and methyl enhance local SOC by two orders of magnitude, fluorine by three orders, and copper enhances local SOC by four orders of magnitude.

There have already been studies constructing model SOC Hamiltonians induced by adatoms in graphene [40,58]. Our approach to the Hamiltonian building is different from those, so it is not surprising that the forms of the Hamiltonians also differ. The analysis of Weeks et al. [40] focuses on heavy adatoms adsorbed in hollow positions interacting with graphene through three outer $p$-shell orbitals of the adatom. The fine structure of these orbitals, due to the intra-atomic spin-orbit coupling, gives rise, via hybridization with carbon orbitals, to the induced SOC of the $\pi$ band of graphene. The procedure to integrate out (downfold) the adatom orbitals starts from a fully functionalized graphene with global $\mathrm{C}_{6 v}$ symmetry-adatoms are occupying each hexagon-but with 
TABLE III. Summary of local SOC strengths for different adelements: hydrogen, fluorine, methyl, and copper [34-37]. Let us emphasize that compared to the referred manuscripts and notation used therein $-\Lambda_{\mathrm{I}}^{\mathrm{A}}, \Lambda_{\mathrm{I}}^{\mathrm{B}}, \Lambda_{\mathrm{PIA}}^{\mathrm{B}}$, and $\Lambda_{\mathrm{R}}$ - we renamed and also properly rescaled their strengths to match the present convention. A translation between the new and old notations is as follows: $\Lambda_{\mathrm{c}}^{\mathrm{YW}}=\Lambda_{\mathrm{I}}^{\mathrm{A}} /(3 \sqrt{3}), \Lambda_{\mathrm{c}}^{\mathrm{ZZ}}=\Lambda_{\mathrm{I}}^{\mathrm{B}} /(3 \sqrt{3})$, $\Lambda_{\mathrm{f}}^{\mathrm{ZZ}}=2 \Lambda_{\mathrm{PIA}}^{\mathrm{B}} / 3$, and $\Lambda_{\mathrm{f}}^{\mathrm{YZ}}=2 \Lambda_{\mathrm{R}} / 3$.

\begin{tabular}{lcccccc}
\hline \hline Adelement/SOC (meV) & $\Lambda_{\mathrm{c}}^{\mathrm{YW}}$ & $\Lambda_{\mathrm{c}}^{\mathrm{ZZ}}$ & $\Lambda_{\mathrm{f}}^{\mathrm{ZZ}}$ & $\Lambda_{\mathrm{f}}^{\mathrm{YZ}}$ & $\Lambda_{\mathrm{f}}^{\mathrm{YY}}$ & $\Lambda_{\mathrm{f}}^{\mathrm{YZ}}$ \\
\hline Hydrogen (top) [34] & -0.04 & - & -0.51 & 0.22 & - & - \\
Fluorine (top) [35] & - & 0.64 & 4.87 & 7.47 & - & - \\
Methyl (top) [36] & -0.15 & 0.03 & -0.46 & 0.68 & - & - \\
Copper (top) [37] & - & 1.73 & 31.6 & 20.1 & - & - \\
Copper (bridge) [37] & - & - & - & - & 41.0 & -7.5 \\
\hline
\end{tabular}

no direct coupling between the orbitals on neighboring adatoms. In contrast, our approach treats a single adatom, so the system has only a local symmetry. Our Hamiltonian for the hollow position thus differs from the one obtained in Ref. [40]. In the work of Pachoud et al. [58], all three relevant adatom configurations are considered, and the choice of the adatom orbitals is not restricted. However, the form of the Hamiltonians is limited to the spatial delta-function (at the adatom site) multiplied by an $8 \times 8$ matrix to cover the pseudospin, valley, and spin spaces. The local structure is thus not preserved, which is not a problem in the continuum limit. Our models instead keep all the local symmetries that adatoms induce (or, rather, still preserve), by assigning pseudospin, spin, and valley-dependent hopping elements in the close neighborhood of the adatom site.

In summary, we have provided in full detail a derivation of effective SOC Hamiltonians for hexagonal systems employing group theory analysis. Our results cover several experimentally relevant scenarios: (i) global SOC effects caused by the proximity of substrates, such as transition metal dichalcogenides, or metallic interfaces; and (ii) local SOC effects due to dilute adatom or admolecule functionalization with emphasis on hollow, top, and bridge adsorption positions. For both cases (i) and (ii), we have explicitly shown which effective SOC matrix elements are suppressed by the presence or absence of particular symmetries and classified the SOC mediated hoppings by the subgroups of the full hexagonal point group. In general, our construction-oriented approach is easily transferable to systems with other symmetries and allows one to derive quickly a particular effective SOC Hamiltonian respecting the given symmetries. Such effective SOC Hamiltonians serve as useful ingredients for model calculations that investigate transport, (quantum) spin Hall effect, spin relaxation and dephasing, WL/WAL measurements, etc.

\section{ACKNOWLEDGMENTS}

This work was supported by DFG SFB 689 and GRK 1570 and by the European Union's Horizon 2020 research and innovation programme under Grant agreement No. 696656.
[1] I. Žutić, J. Fabian, and S. Das Sarma, Rev. Mod. Phys. 76, 323 (2004).

[2] J. Fabian, A. Matos-Abiague, C. Ertler, P. Stano, and I. Žutić, Acta Phys. Slovaca 57, 565 (2007).

[3] W. Han, R. K. Kawakami, M. Gmitra, and J. Fabian, Nat. Nano 9, 794 (2014).

[4] K. S. Novoselov, A. K. Geim, S. V. Morozov, D. Jiang, Y. Zhang, S. V. Dubonos, I. V. Grigorieva, and A. A. Firsov, Science 306, 666 (2004).

[5] C. Lee, X. Wei, J. W. Kysar, and J. Hone, Science 321, 385 (2008).

[6] P. R. Wallace, Phys. Rev. 71, 622 (1947).

[7] M. I. Katsnelson, K. S. Novoselov, and A. K. Geim, Nat. Phys. 2, 620 (2006).

[8] R. V. Gorbachev, A. S. Mayorov, A. K. Savchenko, D. W. Horsell, and F. Guinea, Nano Lett. 8, 1995 (2008).

[9] M. Gmitra, S. Konschuh, C. Ertler, C. Ambrosch-Draxl, and J. Fabian, Phys. Rev. B 80, 235431 (2009).

[10] D. Huertas-Hernando, F. Guinea, and A. Brataas, Phys. Rev. B 74, 155426 (2006).

[11] C. Ertler, S. Konschuh, M. Gmitra, and J. Fabian, Phys. Rev. B 80, 041405 (2009).
[12] B. Dóra, F. Murányi, and F. Simon, Eur. Phys. Lett. 92, 17002 (2010).

[13] J.-S. Jeong, J. Shin, and H.-W. Lee, Phys. Rev. B 84, 195457 (2011).

[14] V. K. Dugaev, E. Y. Sherman, and J. Barnaś, Phys. Rev. B 83, 085306 (2011).

[15] N. Tombros, C. Jozsa, M. Popinciuc, H. T. Jonkman, and B. J. van Wees, Nature (London) 448, 571 (2007).

[16] N. Tombros, S. Tanabe, A. Veligura, C. Jozsa, M. Popinciuc, H. T. Jonkman, and B. J. van Wees, Phys. Rev. Lett. 101, 046601 (2008).

[17] K. Pi, W. Han, K. M. McCreary, A. G. Swartz, Y. Li, and R. K. Kawakami, Phys. Rev. Lett. 104, 187201 (2010).

[18] W. Han and R. K. Kawakami, Phys. Rev. Lett. 107, 047207 (2011).

[19] A. Avsar, T.-Y. Yang, S. Bae, J. Balakrishnan, F. Volmer, M. Jaiswal, Z. Yi, S. R. Ali, G. Güntherodt, B. H. Hong et al., Nano Lett. 11, 2363 (2011).

[20] S. Jo, D.-K. Ki, D. Jeong, H.-J. Lee, and S. Kettemann, Phys. Rev. B 84, 075453 (2011).

[21] R. G. Mani, J. Hankinson, C. Berger, and W. A. de Heer, Nat. Commun. 3, 996 (2012). 
[22] D. Kochan, M. Gmitra, and J. Fabian, Phys. Rev. Lett. 112, 116602 (2014).

[23] D. Kochan, M. Gmitra, and J. Fabian, in Symmetry, Spin Dynamics and the Properties of Nanostructures, Lecture Notes of the 11th International School on Theoretical Physics (World Scientific Singapore, 2015), p. 136.

[24] D. Kochan, S. Irmer, M. Gmitra, and J. Fabian, Phys. Rev. Lett. 115, 196601 (2015)

[25] T. O. Wehling, S. Yuan, A. I. Lichtenstein, A. K. Geim, and M. I. Katsnelson, Phys. Rev. Lett. 105, 056802 (2010).

[26] O. V. Yazyev, Phys. Rev. Lett. 101, 037203 (2008).

[27] V. M. Pereira, F. Guinea, J. M. B. Lopes dos Santos, N. M. R. Peres, and A. H. Castro Neto, Phys. Rev. Lett. 96, 036801 (2006).

[28] D. Soriano, D. V. Tuan, S. M.-M. Dubois, M. Gmitra, A. W. Cummings, D. Kochan, F. Ortmann, J.-C. Charlier, J. Fabian, and S. Roche, 2D Mater. 2, 022002 (2015).

[29] M. R. Thomsen, M. M. Ervasti, A. Harju, and T. G. Pedersen, Phys. Rev. B 92, 195408 (2015).

[30] J. Wilhelm, M. Walz, and F. Evers, Phys. Rev. B 92, 014405 (2015).

[31] B. Raes, J. E. Scheerder, M. V. Costache, F. Bonell, J. F. Sierra, J. Cuppens, J. V. de Vondel, and S. O. Valenzuela, Nat. Commun. 7, 11444 (2016).

[32] C. L. Kane and E. J. Mele, Phys. Rev. Lett. 95, 226801 (2005).

[33] A. H. Castro Neto and F. Guinea, Phys. Rev. Lett. 103, 026804 (2009).

[34] M. Gmitra, D. Kochan, and J. Fabian, Phys. Rev. Lett. 110, 246602 (2013).

[35] S. Irmer, T. Frank, S. Putz, M. Gmitra, D. Kochan, and J. Fabian, Phys. Rev. B 91, 115141 (2015).

[36] K. Zollner, T. Frank, S. Irmer, M. Gmitra, D. Kochan, and J. Fabian, Phys. Rev. B 93, 045423 (2016).

[37] T. Frank, S. Irmer, M. Gmitra, D. Kochan, and J. Fabian, Phys. Rev. B 95, 035402 (2017).

[38] J. Balakrishnan, G. K. W. Koon, M. Jaiswal, A. H. Castro Neto, and B. Özyilmaz, Nat. Phys. 9, 284 (2013).

[39] J. Balakrishnan, G. K. W. Koon, A. Avsar, Y. Ho, J. H. Lee, M. Jaiswal, S.-J. Baeck, J.-H. Ahn, A. Ferreira, M. A. Cazalilla et al., Nat. Commun. 5, 4748 (2014).

[40] C. Weeks, J. Hu, J. Alicea, M. Franz, and R. Wu, Phys. Rev. X 1, 021001 (2011)

[41] D. Ma, Z. Li, and Z. Yang, Carbon 50, 297 (2012).

[42] J. Hu, J. Alicea, R. Wu, and M. Franz, Phys. Rev. Lett. 109, 266801 (2012).

[43] C. M. Acosta, M. P. Lima, R. H. Miwa, A. J. R. da Silva, and A. Fazzio, Phys. Rev. B 89, 155438 (2014).

[44] S. H. Jhang, M. Marganska, Y. Skourski, D. Preusche, B. Witkamp, M. Grifoni, H. van der Zant, J. Wosnitza, and C. Strunk, Phys. Rev. B 82, 041404 (2010).

[45] M. del Valle, M. Margańska, and M. Grifoni, Phys. Rev. B 84, 165427 (2011).

[46] A. K. Geim and I. V. Grigorieva, Nature (London) 499, 419 (2013).

[47] T. Frank, M. Gmitra, and J. Fabian, Phys. Rev. B 93, 155142 (2016).

[48] A. Varykhalov, J. Sánchez-Barriga, A. M. Shikin, C. Biswas, E. Vescovo, A. Rybkin, D. Marchenko, and O. Rader, Phys. Rev. Lett. 101, 157601 (2008).
[49] A. M. Shikin, A. G. Rybkin, D. Marchenko, A. A. Rybkina, M. R. Scholz, O. Rader, and A. Varykhalov, New J. Phys. 15, 013016 (2013).

[50] T. P. Kaloni, L. Kou, T. Frauenheim, and U. Schwingenschlögl, Appl. Phys. Lett. 105, 233112 (2014).

[51] A. Avsar, J. Y. Tan, T. Taychatanapat, J. Balakrishnan, G. Koon, Y. Yeo, J. Lahiri, A. Carvalho, A. S. Rodin, E. O'Farrell et al., Nat. Commun. 5, 4875 (2014).

[52] Z. Wang, D.-K. Ki, H. Chen, H. Berger, A. H. MacDonald, and A. F. Morpurgo, Nat. Commun. 6, 8339 (2015).

[53] M. Gmitra, D. Kochan, P. Högl, and J. Fabian, Phys. Rev. B 93, 155104 (2016)

[54] T. Stauber and J. Schliemann, New J. Phys. 11, 115003 (2009).

[55] M.-H. Liu, J. Bundesmann, and K. Richter, Phys. Rev. B 85, 085406 (2012).

[56] A. Scholz, T. Stauber, and J. Schliemann, Phys. Rev. B 86, 195424 (2012)

[57] A. Ferreira, T. G. Rappoport, M. A. Cazalilla, and A. H. Castro Neto, Phys. Rev. Lett. 112, 066601 (2014).

[58] A. Pachoud, A. Ferreira, B. Özyilmaz, and A. H. Castro Neto, Phys. Rev. B 90, 035444 (2014).

[59] D. V. Tuan, F. Ortmann, D. Soriano, S. O. Valenzuela, and S. Roche, Nat. Phys. 10, 857 (2014).

[60] J. Bundesmann, D. Kochan, F. Tkatschenko, J. Fabian, and K. Richter, Phys. Rev. B 92, 081403 (2015).

[61] M.-H. Liu, P. Rickhaus, P. Makk, E. Tóvári, R. Maurand, F. Tkatschenko, M. Weiss, C. Schönenberger, and K. Richter, Phys. Rev. Lett. 114, 036601 (2015).

[62] J. H. Garcia and T. G. Rappoport, 2D Mater. 3, 024007 (2016).

[63] A. W. Cummings and S. Roche, Phys. Rev. Lett. 116, 086602 (2016).

[64] P.-H. Chang, M. S. Bahramy, N. Nagaosa, and B. K. Nikolić, Nano Lett. 14, 3779 (2014).

[65] D. Van Tuan and S. Roche, Phys. Rev. Lett. 116, 106601 (2016).

[66] D. Van Tuan, J. M. Marmolejo-Tejada, X. Waintal, B. K. Nikolić, S. O. Valenzuela, and S. Roche, Phys. Rev. Lett. 117, 176602 (2016).

[67] H.-Y. Yang, C. Huang, H. Ochoa, and M. A. Cazalilla, Phys. Rev. B 93, 085418 (2016).

[68] C. Huang, Y. D. Chong, and M. A. Cazalilla, Phys. Rev. B 94, 085414 (2016).

[69] L. Brey, Phys. Rev. B 92, 235444 (2015).

[70] G. F. Koster, J. O. Dimmock, R. G. Wheeler, and H. Statz, Properties of the Thirty-Two Point Groups (MIT Press, Cambridge, Massachusetts, 1963).

[71] M. S. Dresselhaus, G. Dresselhaus, and A. Jorio, Group Theory: Application to the Physics of Condensed Matter (SpringerVerlag, Berlin Heidelberg, 2008).

[72] J. C. Slonczewski, PhD Thesis, Rurgers University, 1955.

[73] G. L. Bir and G. E. Pikus, Symmetry and Strain Induced Effects in Semiconductors (Wiley, New York, 1974).

[74] R. Winkler, Spin-Orbit Coupling Effects in Two-Dimensional Electron and Hole Systems (Springer, Berlin, 2003).

[75] S. Konschuh, M. Gmitra, and J. Fabian, Phys. Rev. B 82, 245412 (2010).

[76] S. Konschuh, PhD Thesis, University of Regensburg, 2011.

[77] C.-C. Liu, H. Jiang, and Y. Yao, Phys. Rev. B 84, 195430 (2011).

[78] F. Geissler, J. C. Budich, and B. Trauzettel, New J. Phys. 15, 085030 (2013).

[79] J. C. Slater and G. F. Koster, Phys. Rev. 94, 1498 (1954). 
[80] P. O. Löwdin, J. Chem. Phys. 19, 1396 (1951).

[81] J. W. McClure and Y. Yafet, in Proceedings of the Fifth Conference on Carbon (Pergamon, New York, 1962), Vol. 1, pp. 22-28.

[82] J. O. Sofo, A. S. Chaudhari, and G. D. Barber, Phys. Rev. B 75, 153401 (2007).

[83] K. Takeda and K. Shiraishi, Phys. Rev. B 50, 14916 (1994).

[84] S. Cahangirov, M. Topsakal, E. Aktürk, H. Şahin, and S. Ciraci, Phys. Rev. Lett. 102, 236804 (2009).

[85] J. Zhou, Q. Wang, Q. Sun, X. S. Chen, Y. Kawazoe, and P. Jena, Nano Lett. 9, 3867 (2009).

[86] S. Konschuh, M. Gmitra, D. Kochan, and J. Fabian, Phys. Rev. B 85, 115423 (2012).

[87] Y. A. Bychkov and E. I. Rashba, Pis'ma Zh. Eksp. Teor. Fiz. 39, 66 (1983) [JETP Lett. 39, 71 (1984)].

[88] C. L. Kane and E. J. Mele, Phys. Rev. Lett. 95, 146802 (2005).

[89] J. P. Robinson, H. Schomerus, L. Oroszlány, and V. I. Fal'ko, Phys. Rev. Lett. 101, 196803 (2008).
[90] K. T. Chan, J. B. Neaton, and M. L. Cohen, Phys. Rev. B 77, 235430 (2008).

[91] Y. Mao, J. Yuan, and J. Zhong, J. Phys.: Condens. Matter 20, 115209 (2008).

[92] O. Leenaerts, B. Partoens, and F. M. Peeters, Phys. Rev. B 77, 125416 (2008).

[93] D. W. Boukhvalov, M. I. Katsnelson, and A. I. Lichtenstein, Phys. Rev. B 77, 035427 (2008).

[94] M. Wu, E.-Z. Liu, and J. Z. Jiang, Appl. Phys. Lett. 93, 082504 (2008).

[95] H. Şahin, M. Topsakal, and S. Ciraci, Phys. Rev. B 83, 115432 (2011).

[96] M. Wu, E.-Z. Liu, M. Y. Ge, and J. Z. Jiang, Appl. Phys. Lett. 94, 102505 (2009).

[97] M. Amft, S. Lebégue, O. Eriksson, and N. V. Skorodumova, J. Phys.: Condens. Matter 23, 395001 (2011).

[98] V. I. Anisimov, J. Zaanen, and O. K. Andersen, Phys. Rev. B 44, 943 (1991). 